\title{
The rumen liquid metatranscriptome of post-weaned dairy calves differed by pre-weaning ruminal administration of differentially-enriched, rumen-derived inocula
}

Tansol Park ${ }^{1,2^{*}+}$, Laura M. Cersosimo ${ }^{1 \dagger}$, Wendy Radloff ${ }^{1}$, Geoffrey I. Zanton ${ }^{1}$ and Wenli Li $^{1^{*}}$

\begin{abstract}
Background: Targeted modification of the dairy calf ruminal microbiome has been attempted through rumen fluid inoculation to alter productive phenotypes later in life. However, sustainable effects of the early life interventions have not been well studied, particularly on the metabolically active rumen microbiota and its functions. This study investigated the sustained effects of adult-derived rumen fluid inoculations in pre-weaning dairy calves on the active ruminal microbiome of post-weaned dairy calves analyzed via RNA-sequencing.

Results: Two different adult-derived microbial inocula (bacterial- or protozoal-enriched rumen fluid; BE or PE, respectively) were administered in pre-weaned calves (3-6 weeks) followed by analyzing active rumen microbiome of post-weaned calves ( 9 weeks). The shared bacterial community at the genus level of 165 amplicon-seq and RNA-seq datasets was significantly different $(P=0.024), 21$ out of 31 shared major bacterial genera differed in their relative abundance between the two analytic pipelines. No significant differences were found in any of the prokaryotic alphaand beta-diversity measurements $(P>0.05)$, except the archaeota that differed for BE based on the Bray-Curtis dissimilarity matrix $(P=0.009)$. Even though the relative abundances of potentially transferred microbial and functional features from the inocula were minor, differentially abundant prokaryotic genera significantly correlated to various fermentation and animal measurements including butyrate proportion, body weight, and papillae length and counts. The overall microbial functions were affected quantitatively by BE and qualitatively by $P E(P<0.05)$, and this might be supported by the individual KEGG module and CAZymes profile differences. Exclusive networks between major active microbial (bacterial and archaeal genera) and functional features (KEGG modules) were determined which were differed by microbial inoculations.

Conclusions: This study demonstrated that actively transcribed microbial and functional features showed reliable connections with different fermentations and animal development responses through adult rumen fluid inoculations compared to our previous $16 \mathrm{~S}$ amplicon sequencing results. Exclusive microbial and functional networks of the
\end{abstract}

\footnotetext{
*Correspondence: tansol1719@gmail.com; Wenli.Li@usda.gov

${ }^{\dagger}$ Tansol Park and Laura M. Cersosimo have contributed equally to this work

1 USDA-Agricultural Research Service, Dairy Forage Research Center, Madison, WI, USA

Full list of author information is available at the end of the article
}

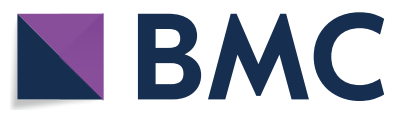

(c) The Author(s) 2021. Open Access This article is licensed under a Creative Commons Attribution 4.0 International License, which permits use, sharing, adaptation, distribution and reproduction in any medium or format, as long as you give appropriate credit to the original author(s) and the source, provide a link to the Creative Commons licence, and indicate if changes were made. The images or other third party material in this article are included in the article's Creative Commons licence, unless indicated otherwise in a credit line to the material. If material is not included in the article's Creative Commons licence and your intended use is not permitted by statutory regulation or exceeds the permitted use, you will need to obtain permission directly from the copyright holder. To view a copy of this licence, visit http://creativecommons.org/licenses/by/4.0/. 
active rumen microbiome of dairy calves created by BE and PE might also be responsible for the different ruminal and animal characteristics. Further understanding of the other parts of the gastrointestinal tract (e.g., abomasum, omasum, and small intestine) using metatranscriptomics will be necessary to elucidate undetermined biological factors affected by microbial inoculations.

Keywords: Adult rumen fluid inoculation, Metatranscriptomics, Protozoa, Dairy calves, Microbiome network

\section{Background}

After raising questions about the difficulties of manipulating the stable and resilient adult rumen microbiota of dairy cattle $[1,2]$, rumen microbiologists have turned attention to targeting the simple and adjustable young dairy calves' microbiota to enhance animal productivity. Starting from the non-functional and sterile gut [3], dairy calves acquire a variety of anaerobic microbiota, including fibrolytic bacteria, particularly following the drastic dietary shift while adapting to solid feeding $[4,5]$. The establishment of adequate multi-kingdom microbiota for dairy calves allows them to use recalcitrant fibers and to maintain the health of the rumen ecosystem $[6,7]$.

Adult-derived rumen microbial inoculation of dairy calves has been studied as a potential probiotic application facilitating the transfer of numerous microbes at once. While adult-rumen microbial inoculation studies with young ruminants didn't significantly impact rumen microbial populations and animal growth measurements $[8,9]$, potential beneficial effects on gut health, fermentations, and rumen development have been demonstrated $[8,10]$.

Previous studies have used DNA amplicon sequencing approaches to describe microbial communities from the rumen contents or rumen epithelium of young ruminants [11-13]. In our previous studies, we investigated the effect of adult-derived rumen fluid inoculation with two different microbial inocula (bacterial- or protozoal-enriched rumen fluid; $\mathrm{BE}$ or $\mathrm{PE}$, respectively) in pre-weaning calves (3-6 weeks) [14] followed by confirmation of the sustained effects of the inoculations in post-weaned calves (9 weeks of age) [15]. Though these studies have shown significant insights into the overall bacterial community structure of the rumen and digestive tract, DNA-based sequencing methods are not capable of measuring the active microbial communities, as they cannot distinguish between inactive, alive, dead, or lysed cells $[16,17]$. In contrast, RNA-based methods enable the study of the structure and potential function of the active microbial community by capturing actively transcribed RNA species. Due to this advantage, mRNA, or total RNA-based sequencing library preparation and cDNA-based, 16S rRNA gene amplicon sequencing (16S amplicon-seq) techniques have been utilized to better understand the active rumen microbial community [17-20].

Few studies have described the transcriptome of rumen fluid from dairy cows. To the author's knowledge, no studies have described the rumen fluid transcriptome of dairy calves dosed with bacterial- and protozoal-enriched rumen inocula. The objectives of our experiment were to use RNA-sequencing to determine if the $\mathrm{BE}$ and $\mathrm{PE}$ microbial inoculations during the pre-weaning period (3-6 weeks of age; [14]) affect the active bacterial and archaeal community structures, associated functional compositions, and their co-occurrence patterns in postweaned dairy calves. As a follow-up to our previously published work, we also compare the results from RNAseq and 16S amplicon-seq based analyses to assess the commonality and uniqueness of both methods.

\section{Methods}

\section{Experimental design and calf management}

The animal procedures, dietary conditions, inoculum preparations, and experimental designs were described in our previous study [15]. Briefly, calves were kept ciliate-free before the experiment by separating them from their dam at birth, received colostrum within $4 \mathrm{~h}$ after birth, and were housed in individual calf hutches at the US Dairy Forage Research Farm in Prairie du Sac, WI. Twenty Holstein bull calves were randomly assigned to one of four different types of ruminal inocula (1. autoclaved, clarified rumen fluid, 2. BE, 3. PE, 4. BE and PE). A detailed description of the collection, processing and separation of ruminal inoculum was detailed in our 2 previous papers $[14,15]$. Briefly, rumen contents from 4 , first lactation, ruminally cannulated cows were removed and either blended, strained, centrifuged, and combined to create the bacteria-enriched supernatant or strained, placed in a separatory funnel for $1 \mathrm{~h}$, and the protozoaenriched pellet was removed and combined to create the protozoa-enriched inoculum. Calves were orally dosed the respective treatment inocula once per week from 3 to 6 weeks of age.

\section{Sample collection}

All the procedures to analyze animal measurements, papillae length and numbers, and rumen fermentation characteristics were measured in our previous study 
at 9 weeks of age [15]. Additionally, calf health (rectal temperatures, nasal discharge, eye, ear, and feces) were assessed at 9 weeks of age using the University of Wisconsin School of Veterinary Medicine's Scoring Chart (https://fyi.extension.wisc.edu/heifermgmt/files/2015/ 02/calf_health_scoring_chart.pdf, accessed January 19, 2021) as previously described [14].

\section{RNA extraction and sequencing}

Rumen fluid samples $(3 \mathrm{~mL}$ ) for RNA analyses were immediately frozen with liquid nitrogen and stored at $-80{ }^{\circ} \mathrm{C}$. Total RNA was extracted from $200 \mu$ frozen rumen fluid (from both the inoculum and rumen samples) using the AllPrep Power Viral Kit (Qiagen, Hildlen, Germany) with the $\beta$-mercaptoethanol and solution PV1 premix option. The RNA integrity number (RIN) was measured with an Agilent 2100 Bioanalyzer. The samples with RIN greater than 7 were pursued for further analysis. RNA sample concentration was checked using a Qbit 3.0 (Thermo Fisher, US). An amount of $500 \mathrm{ng}$ of total RNA from each sample was used for RNA sequencing library preparation using the Illumina TruSeq mRNA kit (Cat. 20020594) and it's protocol (TruSeq stranded mRNA reference guide 1000000040498, Illumina, US). Initial steps of mRNA enrichment (as described under the section of "Purify mRNA", using Poly-T oligo attached magnetic beads) were skipped to maximize the capture of microbial RNA reads. The work flow started by combining $5 \mu \mathrm{l}$ total RNA with the $13 \mu \mathrm{l}$ of Fragment, Prime, Finish mix and continued with the Elution 2-Frag-Prime program on the thermal cycler as described in the protocol and the steps afterwards. The fragment size and distribution of prepared libraries were assessed using a Bioanalyzer DNA 1000 kit (Agilent Technologies, CA, US). Library pooling was done by the library concentration determined by a Kapa quant kit (Cat. KK4873). After initial pooling, a MiSeq run was done using a MiSeq nano kit (Cat. MS-103-1001). Further normalization of the library pooling was done using the index ratio obtained for each library calculated by the MiSeq run. After this step, the final, pooled libraries were sequenced on a NextSeq sequencer using high-output 150-cycle kits (Cat. 20024907) to generate $2 \times 75$ bp paired-end reads.

\section{Metatranscriptome analysis}

Additional file 1: Fig. S1 provided the major analytic workflow for metatranscriptomics used in this study. The quality of RNA-seq raw reads was first checked using FastQC (https://www.bioinformatics.babraham.ac.uk/ projects/fastqc/, accessed on 01/25/2019). To filter out the reads from the host, raw RNA-seq were first mapped to the host reference genome UMD 3.1 of Bos taurus (http://ccb.jhu.edu/software/tophat/igenomes.shtml,
April 1st, 2019) using the STAR aligner [21]. Unmapped reads were considered to be of rumen microbial origin and used for the downstream microbial community analysis. The host genome-filtered reads were used for bacterial and archaeal taxonomic classification using Kraken2 (v.2.0.8-beta) [22] with a confidence score of 0.2. Kraken2 standard database was downloaded and built on 4th June 2021. Among the host genome-filtered reads, non-rRNA reads were separated from rRNA reads by SortMeRNA (version, 2.1b) [23] based on the reference rRNA databases provided by Silva (release 138) [24]. Sequences classified as chloroplast and mitochondria were excluded before downstream analysis. Raw-read counts at each taxonomic level (i.e., phylum and genus) were normalized per sample with the per million factor as described in [25]. Major classified taxa, defined as occupying over $0.01 \%$ average relative abundance in at least one of the treatments, were discussed in this study.

Alpha-diversity measurements including richness indices (number of observed genera and Chaol richness estimates), Evenness, Shannon's index, and Simpson's index were analyzed using the rarefied read count table of classified bacterial and archaeal genera. The overall bacterial and archaeal communities shaped by microbial inoculations were visualized using principal coordinates analysis based on the Bray-Curtis and Jaccard distance matrices at the genus level.

FastSpar [26], which uses the SparCC algorithm to calculate correlation estimations for compositional data [27], analyzed co-occurrence and mutual exclusion networks among the major microbial and functional features $(>0.01 \%$ relative abundance in at least one of the inoculation conditions) represented by bacterial and archaeal genera and Kyoto Encyclopedia of Genes and Genomes (KEGG) modules, respectively. By comparing the significant interactions between the microbial inoculation treatments and their corresponded controls, exclusive features and interactions were selected using the $R$ package, Co-expression Differential Network Analysis (CoDiNA) [28] and were visualized using Gephi [29].

Venn diagrams were used to visualize the number of shared and exclusively detected bacterial and archaeal genera, and KEGG modules between two types of microbial inoculums and their corresponding rumen liquid samples.

\section{Taxonomic analysis of $16 \mathrm{~S}$ amplicon-seq dataset}

To compare the taxonomic classification outputs between DNA-based and RNA-based datasets, previously published $16 \mathrm{~S}$ amplicon-sequences [15] were reclassified against the Kraken reference dataset. First, amplicon sequence variants generated by DADA2 [30] from the previous study were classified using Kraken2 
and the classification was subsequently transformed to QIIME2-readible taxonomy file. The newly curated taxonomy file was used to re-calculate the abundance of each taxon in each sample. Only the major classified phyla and genera (defined as occupying over $0.01 \%$ average relative abundance in at least one of the treatments), which were jointly found in both the $16 \mathrm{~S}$ amplicon-seq and RNA-seq datasets, were further analyzed for both the individual taxa and overall bacterial communities at genus level between those datasets.

\section{Functional annotation based on the non-rRNAs}

Non-rRNA reads, which were the remainders after filtering out the sequences mapped to the bovine genome and rRNA genes, were considered as microbial protein coding gene reads and were used to analyze the potential microbial functions. Following the analytic pipeline suggested in Dai et al. [31], non-rRNAs were searched against NCBI nonredundant protein sequences (downloaded and built on 20th May 2020) using blastx module in DIAMOND [32] with an E-value of $1 \mathrm{e}-5$ and a bit score of 50 as a cutoff. The deduced protein sequences were extracted from best-hit and used to annotate KEGG ortholog profiles via the KEGG Automatic Annotation Server (KAAS) [33] using GHOSTX program to search prokaryotic representative gene sets and subsequently mapped to KEGG modules using the python script implemented in picrust2 package [34]. Carbohydrate-active enzymes (CAZymes) annotations were further searched using non-rRNAs of inoculum and rumen samples by dbCAN2 [35]. The CAZymes were reported when at least two out of three annotation tools [i.e., HMMER (http://www.hmmer.org), DIAMOND, and Hotpep [36]] detected the same annotations satisfying recommended search cutoffs. The relative abundance of each CAZyme was calculated based on the total number of annotated CAZymes.

\section{Statistical analysis}

Inoculum treatments were denoted as follows; with or without $\mathrm{BE}$ inoculation: $\mathrm{BE}(+)$ or $\mathrm{BE}(-)$, respectively, and with or without $\mathrm{PE}$ inoculation: $\mathrm{PE}(+)$ or $\mathrm{PE}(-)$, respectively. Comparing bacterial and archaeal alpha-diversity measurements and the abundance of CAZymes between two types of the inoculums was done with PROC GLIMMIX procedure in SAS 9.3 (SAS Institute Inc., Cary, NC, USA) with the fixed effect of inoculum type and random effect of calf. Negative bionomial distribution was applied to the features with non-normal distribution. Effects of microbial inoculations (BE or PE) on the alpha-diversity measurements at genus level were statistically analyzed using the GLIMMIX procedure of SAS 9.3 and the model included fixed effects of $\mathrm{BE}$ and $\mathrm{PE}$, and the interaction between $\mathrm{BE}$ and PE. LEfSe [linear discriminant analysis
(LDA) effect size] [37] was used to examine the difference of normalized bacterial and archaeal abundances at both phylum and genus levels and normalized counts of KEGG modules and annotated CAZymes by microbial inoculations with an LDA score 2 as cutoff. Calf health assessments were also analyzed using LEfSe except rectal temperature. Permutational multivariate analysis of variance (PERMANOVA) analysis (vegan::Adonis with 9,999 random permutations) was computed to analyze the beta-diversity based on the Bray-Curtis and Jaccard distance matrices of bacteriota, archaeota and functional profiles of KEGG modules. The correlations of normalized abundance of major active bacterial and archaeal genera with the animal performance measurements, fermentation characteristics, and protozoal counts which have been previously reported in Park et al. [15] were determined based on the Pearson correlation coefficients (correlation coefficient, $|r| \geq 0.6, P \leq 0.05)$ using PROC CORR procedure in SAS 9.3 and subsequently visualized using the corrplot package in $\mathrm{R}$ (v3.5.0). Within each CoDiNA selected exclusive network, keystone microbial and functional nodes were chosen based on two of the centrality measurements (i.e., authority and eigenvector centrality) calculated using the built-in plugins implemented in Gephi [29]. Significance was declared at $P \leq 0.05$ and trends at $0.05<P \leq 0.1$.

\section{Results}

\section{Calf health assessments}

All the assessments including rectal temperatures, nasal discharges, eye, ear, respiratory and fecal scores of dairy calves were not significantly differed by $\mathrm{BE}$ and $\mathrm{PE}$ inoculations $(P>0.05)$ (Additional file 2: Table S1).

\section{Comparative analysis of rumen bacteria using RNA-seq and $16 \mathrm{~S}$ amplicon-seq}

In total, there were 37 and 13 bacterial phyla and 1,207 and 106 bacterial genera classified from the RNA-seq and $16 \mathrm{~S}$ amplicon-seq, respectively. Among the nine major bacterial phyla (defined as occupying over $0.01 \%$ average relative abundance in at least one of the analytic pipelines) detected by both of the RNA-seq and $16 \mathrm{~S}$ amplicon-seq analytic pipelines in the rumen fluid samples of dairy calves, the relative abundance of Bacteroidetes was greater in $16 \mathrm{~S}$ amplicon-seq dataset whereas the relative abundance of six phyla (Elusimicrobia, Fibrobacteres, Firmicutes, Planctomycetes, Spirochaetes, and Verrucomicrobia) were greater in RNA-seq dataset (Fig. 1). At genus level, 31 shared major bacterial genera represented 21.2 and $43.6 \%$ of the total bacteriota which were analyzed by RNA-seq and $16 \mathrm{~S}$ amplicon-seq, respectively. Meanwhile, 1150 genera representing about $2.34 \%$ of overall bacteriota 


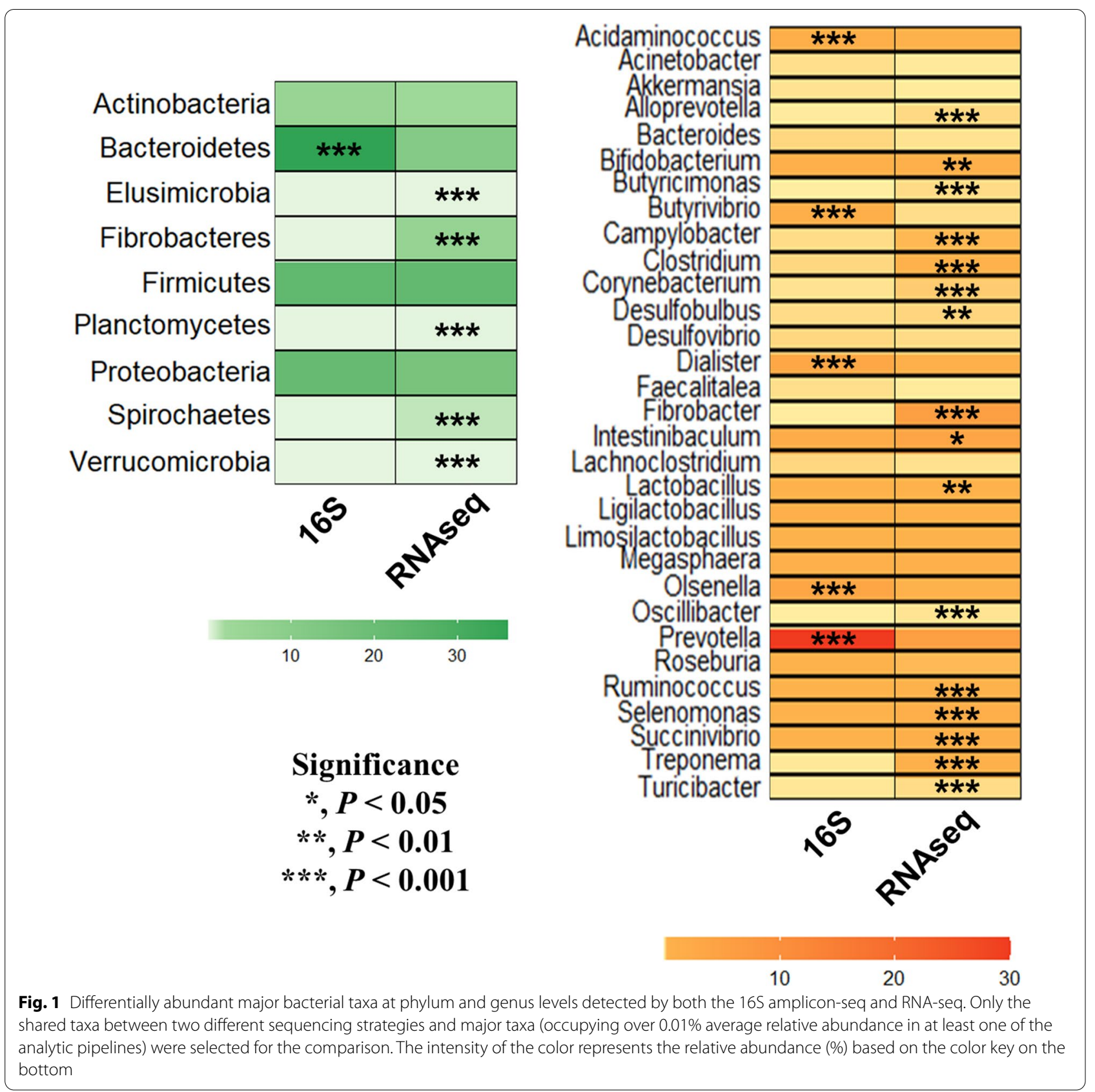

were uniquely identified in RNA-seq and 22 of those (Lancefieldella, Collinsella, Berryella, Duncaniella, Sodaliphilus, Rufibacter, Cruoricaptor, Solitalea, Elusimicrobium, Bacillus, Staphylococcus, Eubacterium, Anaerostipes, Blautia, Coprococcus, Bulleidia, Planctopirus, Solimonas, Moraxella, Vibrio, Sphaerochaeta, and Ereboglobus) were considered as major genera in active bacteriota, whereas no classified genera were uniquely identified in $16 \mathrm{~S}$ amplicon-seq (data not shown). Except 10 genera (Acinetobacter, Akkermansia, Bacteroides, Desulfovibrio, Faecalitalea, Lachnoclostridium, Ligilactobacillus, Limosilactobacillus, Megasphaera and Roseburia), 21 out of 31 genera differed in their relative abundances between two analytic pipelines. Based on the Bray-Curtis dissimilarity matrices, the shared bacterial community at the genus level of $16 \mathrm{~S}$ amplicon-seq and RNA-seq datasets was significantly different $(P=0.024)$ (Additional file 3 : Fig. S2) and the overall bacterial community (based on 
Bray-Curtis distance matrix) between the two analytic pipelines were also significantly different $(P=0.027)$ (data not shown).

\section{The impact of adult rumen inoculation on the rumen bacterial diversity, archaeal diversity, and overall functional distribution}

From the extracted RNA samples, with the average RNA integrity number of $7.4 \pm 0.25$ [(mean \pm standard error of the mean (SEM)], an average of 59,153,238 $\pm 1,314,352$ high-quality reads were generated with a range of 48,091,338 to 70,643,716 reads (Additional file 2: Table S2). For the inoculum samples, an average of $51,733,503 \pm 5,150,354$ high-quality reads were generated with a range of $42,515,561$ to $61,110,565$ reads (Additional file 2: Table S3). Following the quality filtering and removal of reads from host genome, $58,890,367 \pm 1,309,652$ host genome-filtered reads per sample were successfully classified. These classified reads comprised at least over $68.6 \%$ of the host genome-filtered reads per sample by Kraken2. Alpha- and beta-diversity analyses were done with rarefied genus level BIOM tables containing 5,333,080 and 584,744 counts for bacteriota and archaeota, respectively. Good's coverage of genus level classification was higher than $99.9 \%$ in all RNA-seq samples.

Two inoculums had no differences for bacterial alphadiversity measurements, whereas archaeal evenness and Shannon's index was significantly higher in bacteriaenriched inoculum than those of the protozoa-enriched inoculum $(P>0.05)$ (Additional file 2: Table S4). No differences were observed for all microbial alpha-diversity measurements by both the microbial inoculations (Table 1). Based on the Bray-Curtis (quantitative) and
Jaccard (qualitative) distance matrices, overall bacterial microbiota did not differ by $\mathrm{BE}$ and $\mathrm{PE}$ inoculations $(P>0.1$; Fig. 2$)$, whereas archaeal microbiota was significantly different between that of $\mathrm{BE}(+)$ and $\mathrm{BE}(-)$ calves based on the quantitative matrix $(P=0.009$; Additional file 4: Fig. S3).

At the phylum level, Proteobacteria was dominant in $\mathrm{PE}(-)$ calves $[\mathrm{PE}(+): 12.980 \pm 1.470 \%$ and $\mathrm{PE}(-)$ : $18.474 \pm 2.327 \%, P=0.049$; Table 2]. Bifidobacterium and two genera within Firmicutes (Anaerostipes and Oscillibacter) were differentially abundant in $\mathrm{BE}(-)$ calves. For PE, the relative abundance of Eubacterium and Desulfobulbus was greater in $\mathrm{PE}(+)$ calves, whereas Roseburia and Megasphaera within Firmicutes and Desulfovibrio and Moraxella within Proteobacteria were dominant in $\mathrm{PE}(-)$ calves.

There were no archaeal phyla and genera differed by either of microbial inoculation treatments. Although not different by microbial inoculation treatments, Methanocaldococcus represented averagely over $60 \%$ of the total archaeal community of dairy calves (data not shown).

Among the protein sequences deduced from the blastx best hit, an average of $49.22 \%$ sequences were mapped to the KEGG orthologs (Additional file 2: Table S2). Based on the KEGG orthologs predicted from the annotation of non-rRNAs, overall microbial functional profiles were affected by microbial inoculations (Fig. 3). BE and PE treatments affected the overall abundance and presence/ absence of microbial functions, respectively $(P<0.05)$. For individual relative abundance of KEGG modules, seven KEGG modules [M00006, pentose phosphate pathway, oxidative phase, glucose $6 \mathrm{P}=>$ ribulose $5 \mathrm{P}(P=0.041)$; M00033, ectoine biosynthesis, aspartate $=>$ ectoine $(P=0.024) ; \quad$ M00140, C1-unit interconversion,

Table 1 Alpha diversity measurements in microbial inoculation treatments

\begin{tabular}{|c|c|c|c|c|c|c|c|c|}
\hline \multirow[t]{2}{*}{ Diversity measurements } & \multicolumn{2}{|c|}{ Bacterial-enriched (BE) } & \multicolumn{2}{|c|}{ Protozoal-enriched (PE) } & \multirow[t]{2}{*}{ SEM } & \multicolumn{3}{|c|}{$P$-values } \\
\hline & + & - & + & - & & BE & PE & $\mathrm{BE} \times \mathrm{PE}$ \\
\hline \multicolumn{9}{|l|}{ Bacterial alpha-diversity } \\
\hline Observed genera & 525 & 507 & 533 & 499 & 17.465 & 0.643 & 0.367 & 0.906 \\
\hline Chao1 estimates & 668 & 663 & 685 & 646 & 22.106 & 0.923 & 0.416 & 0.762 \\
\hline Evenness & 0.328 & 0.360 & 0.331 & 0.356 & 0.011 & 0.155 & 0.265 & 0.660 \\
\hline Shannon's index & 2.956 & 3.228 & 2.994 & 3.190 & 0.101 & 0.198 & 0.349 & 0.641 \\
\hline Simpson's index & 0.756 & 0.810 & 0.762 & 0.804 & 0.019 & 0.161 & 0.279 & 0.690 \\
\hline \multicolumn{9}{|l|}{ Archaeal alpha-diversity } \\
\hline Observed genera & 11 & 10 & 11 & 9 & 0.505 & 0.295 & 0.219 & 0.923 \\
\hline Chao1 estimates & 12 & 10 & 12 & 10 & 0.827 & 0.306 & 0.306 & 0.808 \\
\hline Evenness & 0.362 & 0.309 & 0.334 & 0.337 & 0.025 & 0.326 & 0.956 & 0.910 \\
\hline Shannon's index & 1.224 & 0.995 & 1.137 & 1.082 & 0.088 & 0.225 & 0.766 & 0.987 \\
\hline Simpson's index & 0.438 & 0.349 & 0.393 & 0.393 & 0.036 & 0.258 & 0.997 & 0.973 \\
\hline
\end{tabular}




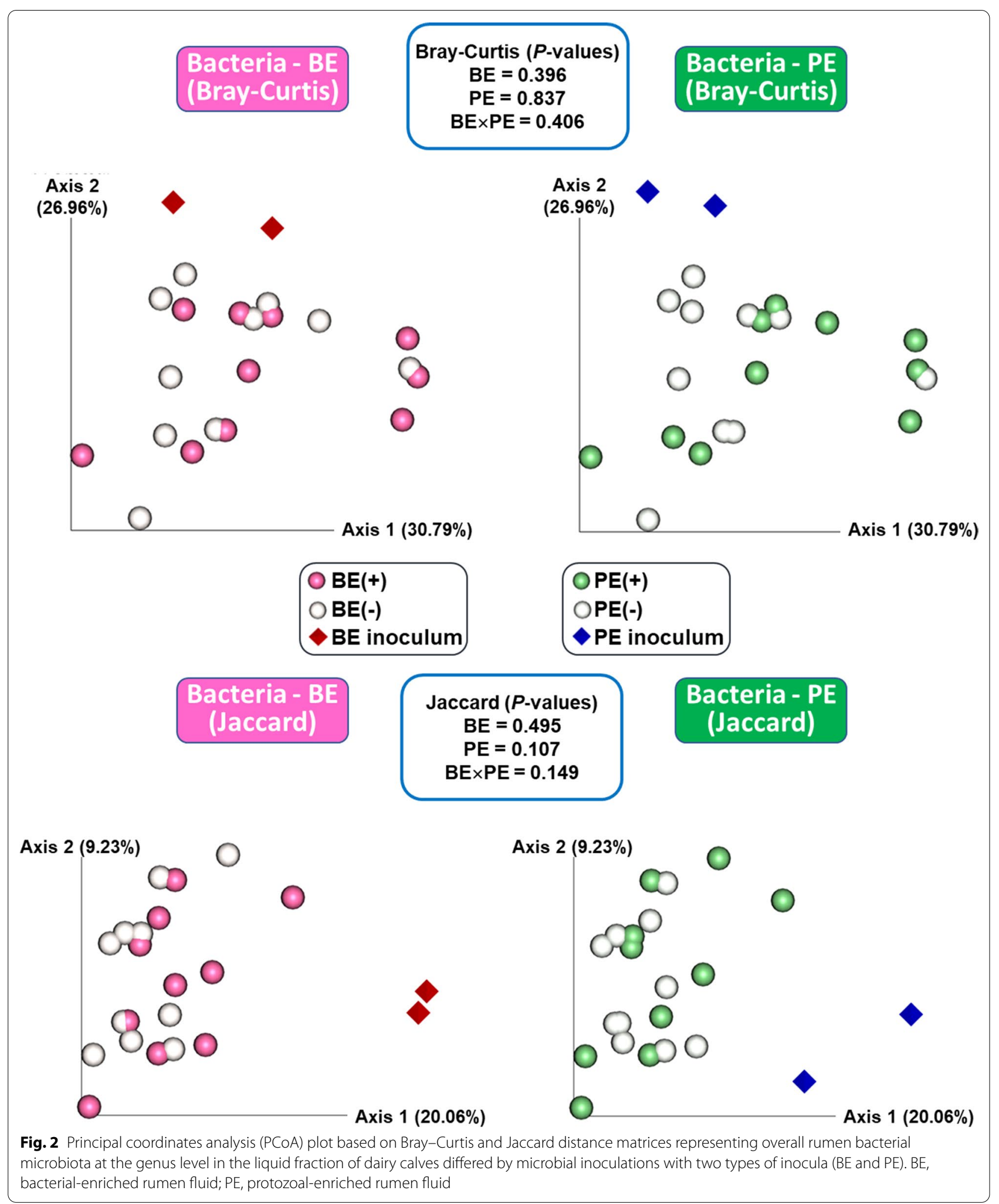


Table 2 Differentially abundant bacterial taxa (phyla and genera) by microbial inoculations

\begin{tabular}{|c|c|c|c|c|c|c|}
\hline \multirow[t]{2}{*}{ Dominance } & \multirow[t]{2}{*}{ Phylum } & \multirow[t]{2}{*}{ Genus } & \multicolumn{2}{|c|}{ Relative abundance (\%) } & \multirow[t]{2}{*}{ SEM } & \multirow[t]{2}{*}{$P$-value } \\
\hline & & & $\mathrm{BE}(+)$ & $\mathrm{BE}(-)$ & & \\
\hline \multicolumn{7}{|c|}{ By bacterial-enriched rumen fluid inoculation } \\
\hline \multirow[t]{3}{*}{$\mathrm{BE}(-)$} & Actinobacteria & Bifidobacterium & 0.550 & 1.015 & 0.157 & 0.041 \\
\hline & Firmicutes & Anaerostipes & 0.001 & 0.018 & 0.006 & 0.005 \\
\hline & & Oscillibacter & 0.008 & 0.012 & 0.002 & 0.049 \\
\hline \multirow[t]{2}{*}{ Dominance } & Phylum & Phylum & \multicolumn{2}{|c|}{ Relative abundance (\%) } & SEM & $P$-value \\
\hline & & & $\mathrm{PE}(+)$ & $\mathrm{PE}(-)$ & & \\
\hline \multicolumn{7}{|c|}{ By protozoal-enriched rumen fluid inoculation } \\
\hline $\mathrm{PE}(-)$ & Proteobacteria & - & 12.980 & 18.474 & 1.480 & 0.049 \\
\hline \multirow[t]{2}{*}{ Dominance } & \multirow[t]{2}{*}{ Phylum } & \multirow[t]{2}{*}{ Genus } & \multicolumn{2}{|c|}{ Relative abundance (\%) } & SEM & $P$-value \\
\hline & & & $\mathrm{PE}(+)$ & $\mathrm{PE}(-)$ & & \\
\hline \multirow[t]{2}{*}{$\mathrm{PE}(+)$} & Firmicutes & Eubacterium & 0.083 & 0.026 & 0.014 & 0.049 \\
\hline & Proteobacteria & Desulfobulbus & 0.037 & 0.012 & 0.005 & 0.019 \\
\hline \multirow[t]{4}{*}{$P E(-)$} & Firmicutes & Roseburia & 0.014 & 0.094 & 0.017 & 0.019 \\
\hline & & Megasphaera & 0.379 & 0.790 & 0.085 & 0.013 \\
\hline & Proteobacteria & Desulfovibrio & 0.009 & 0.029 & 0.007 & 0.007 \\
\hline & & Moraxella & 0.282 & 0.453 & 0.039 & 0.041 \\
\hline
\end{tabular}

Only the taxa which have over $0.01 \%$ average relative abundance in at least one of the treatments $[\mathrm{BE}(+), \mathrm{BE}(-), \operatorname{PE}(+), \operatorname{PE}(-)]$ were statistically analyzed by $L E f S e$ $\mathrm{BE}$, bacterial-enriched rumen fluid; $\mathrm{PE}$, protozoal-enriched rumen fluid

${ }^{*}$ Calculated based on the normalized read count BIOM tables

prokaryotes $(P=0.041) ;$ M00166, reductive pentose phosphate cycle, ribulose- $5 \mathrm{P}=>$ glyceraldehyde- $3 \mathrm{P}$ $(P=0.025) ;$ M00299, spermidine/putrescine transport system $(P=0.028)$; M00314, bacitracin transport system $(P=0.042)$; and M00368, ethylene biosynthesis, methionine $=>$ ethylene $(P=0.013)]$ were differentially enriched in $\mathrm{BE}(+)$ calves rather than $\mathrm{BE}(-)$ calves (Fig. 4). The relative abundance of M00015 [Proline biosynthesis, glutamate $=>$ proline $(P=0.034))$, M00020 [serine biosynthesis, glycerate-3P $=>$ serine $(P=0.019)]$, and M00299 [spermidine/putrescine transport system $(P=0.034)$ ] were greater in $\mathrm{PE}(+)$ calves compared to that of $\mathrm{PE}(-)$ calves.

\section{Adult rumen fluid inoculation effects on the presence/ absence of rumen microbial and functional features}

The Venn diagrams showed the number of shared or exclusive bacterial and archaeal taxa at genus level and KEGG modules between microbial inoculation treatments with their corresponding inocula (Fig. 5). Among the number of classified archaeal genera, $31.58 \%$ of total archaeal genera were shared among the BE-inoculum, $\mathrm{BE}(+)$, and $\mathrm{BE}(-)$ and $28.95 \%$ of total archaeal genera were exclusively found in one of the three conditions. Among the number of classified bacterial genera, $60.20 \%$ of total bacterial genera were shared among the three conditions by $\mathrm{BE}$ inoculation and $18.40 \%$ were exclusively found in one of the three conditions. Among 306 KEGG modules, 253 were shared by the three conditions by $\mathrm{BE}$ inoculation and only 19 KEGG modules were exclusively found in one of the three conditions. By PE inoculation and its inoculum, $50.46 \%$ and $30.77 \%$ of bacterial and archaeal genera were shared among PE-inoculum, $\mathrm{PE}(+)$, and $\mathrm{PE}(-)$, respectively. Otherwise, $25.73 \%$ and $35.90 \%$ of bacterial and archaeal genera were exclusively found either in PE-inoculum, $\mathrm{PE}(+)$, and $\mathrm{PE}(-)$, respectively. Among the identified 304 KEGG modules, 245 were shared by the three conditions by PE inoculation and only 20 KEGG modules were exclusively found in one of the three conditions. Both bacterial and archaeal genera, and their identified microbial functions represented by KEGG modules potentially transferred from inocula to inocula-treated calves only occupied minor abundance in both the inoculations $(<0.0001 \%$ of bacterial and archaeal abundances whereas $<0.004 \%$ of KEGG modules). In both of the inoculations, the inocula shared a numerically greater number of archaeal and bacterial genera, and KEGG modules with inoculum-treated calves compared to that of non-treated calves. 


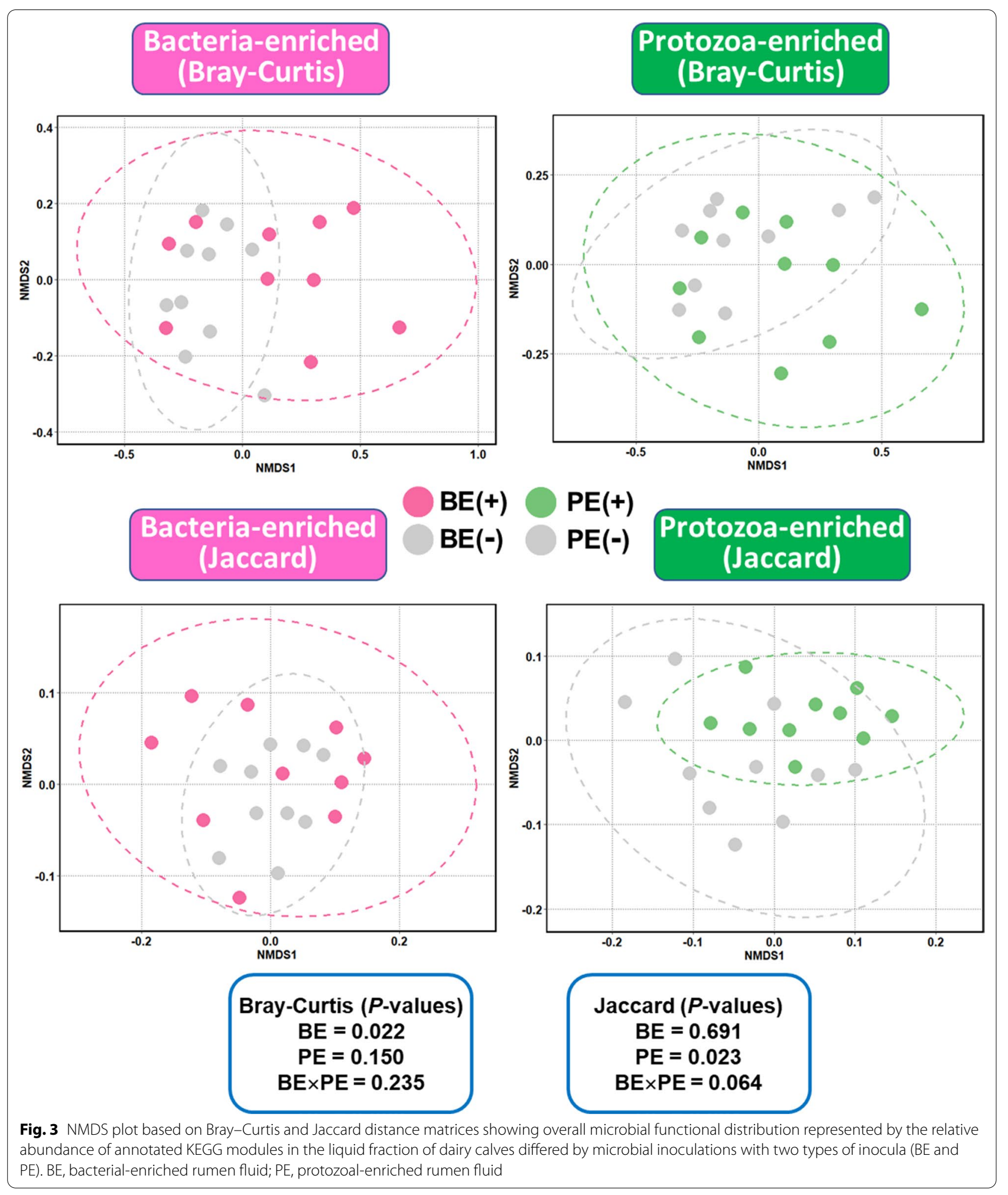

Changes in the co-occurrence or mutual-exclusive microbiome networks of rumen after adult rumen fluid inoculation
The complexed co-occurrence and mutual exclusive interactions among the abundance of major bacterial and archaeal genera and KEGG modules differed by 


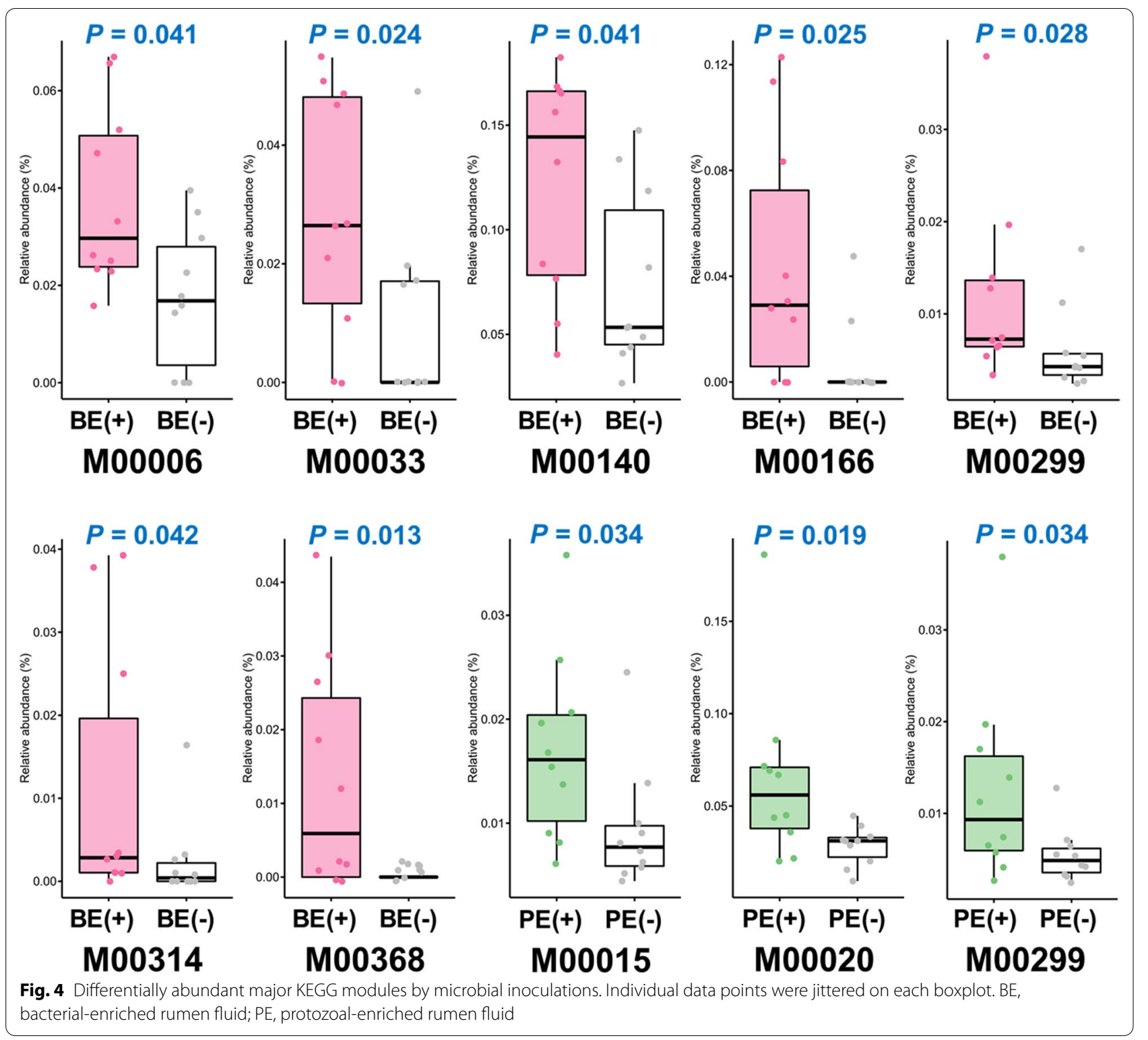

microbial inoculations (Additional file 5: Fig. S4). By BE, 99 (70 KEGG modules, four archaeal genera, and 25 bacterial genera) and 22 (13 KEGG modules, one archaeal genus, and eight bacterial genera) nodes with 260 and 123 significant interactions were exclusively found in $\mathrm{BE}(+)$ and $\mathrm{BE}(-)$ treated calves, respectively (Additional file 5: Fig. S4). A total of 37 (27 KEGG modules, two archaeal genera, and eight bacterial genera) and 71 (48 KEGG modules, two archaeal genera, and 21 bacterial genera) nodes with 199 and 194 significant interactions were exclusively found in $\mathrm{PE}(+)$ and $\mathrm{PE}(-)$ calves, respectively. Based on the two centrality measurements within the exclusive interactions in each inoculation treatment, Prevotella and M00022
(Shikimate pathway, phosphoenolpyruvate + erythrose$4 \mathrm{P}=>$ chorismate) for $\mathrm{BE}(+)$ calves whereas Sodaliphilus and M00184 (RNA polymerase, archaea) for $\mathrm{BE}(-)$ calves were identified as keystone microbial and functional nodes by BE inoculation (Table 3). By PE inoculation, Desulfobulbus and M00049 (Adenine ribonucleotide biosynthesis, IMP $=>$ ADP,ATP) for $\mathrm{PE}(+)$ calves whereas Ligilactobacillus and M00014 [Glucuronate pathway (uronate pathway)) for $\mathrm{PE}(-)$ were the keystone nodes. The interactions centered on those keystone nodes were further selected and visualized in Fig. 6. 


\section{Bacteria-enriched}
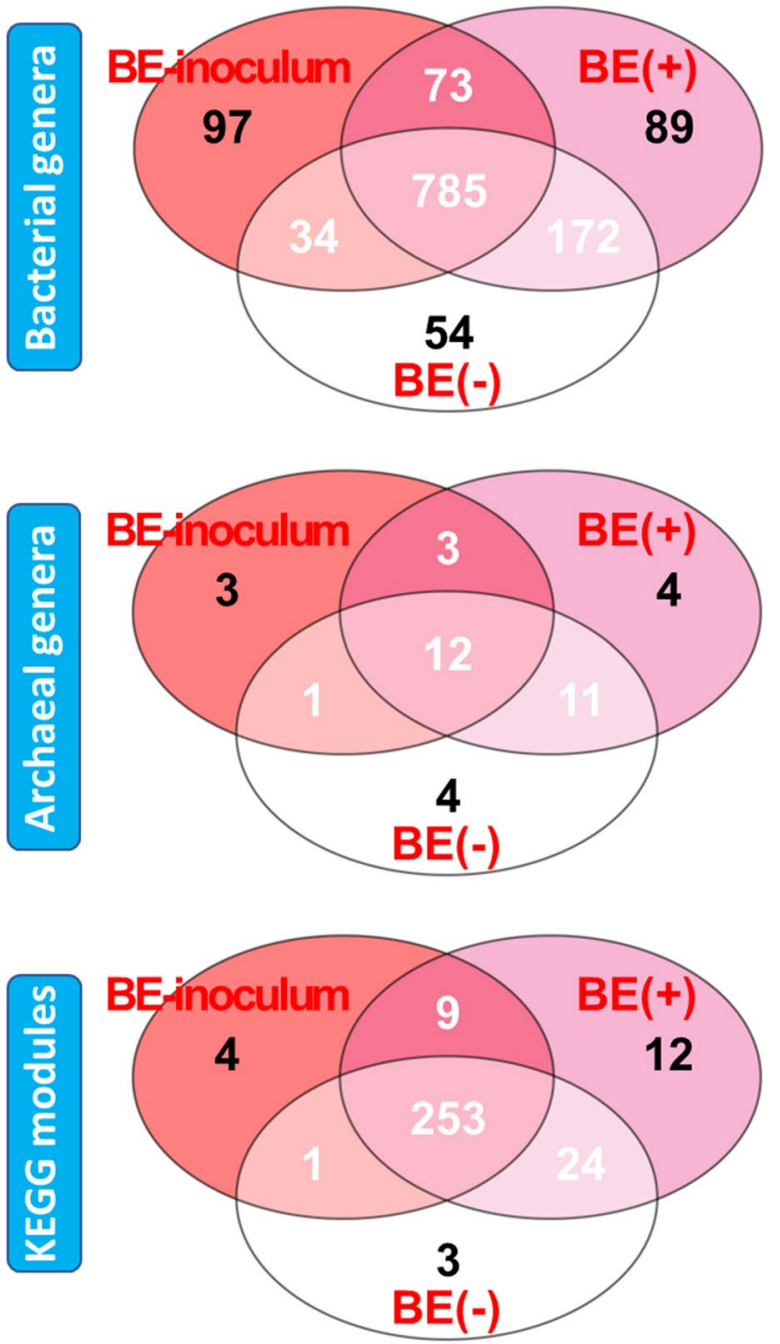
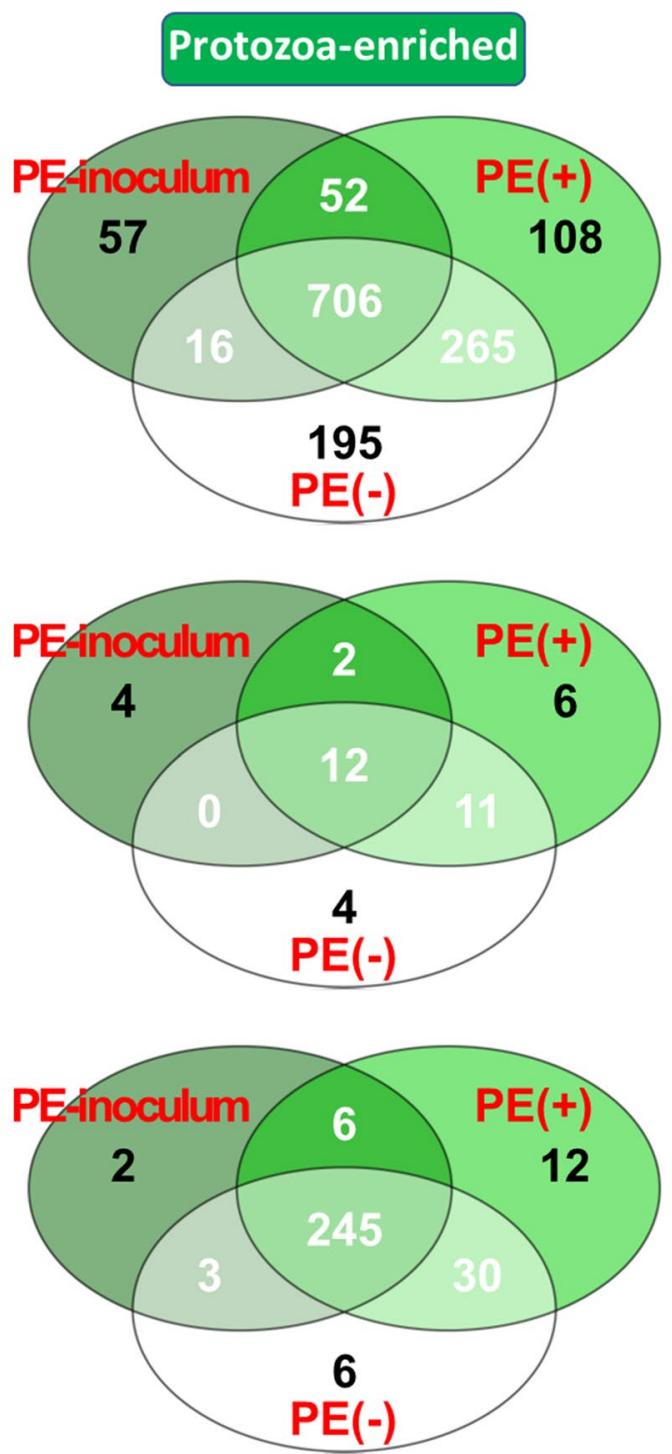

Fig. 5 Number of bacterial, archaeal genera and KEGG modules which were differentially observed by microbial inoculations. The number of shared or exclusively found genera or functional features among the corresponded microbial inocula, inocula-treated and controls were shown in Venn diagram. BE, bacterial-enriched rumen fluid; PE, protozoal-enriched rumen fluid

\section{Differed CAZymes profiles by microbial inoculations}

A total of 186 and 163 CAZymes, belonging to auxiliary activities, carbohydrate-binding modules (CBM), carbohydrate esterases (CE), glycosyl hydrolases $(\mathrm{GH})$, glycosyl transferases (GT), and polysaccharide lyases $(\mathrm{PL})$, were annotated from the BE and PE inoculum, respectively, and there were no differentially abundant CAZymes between BE and PE inoculum (data not shown).

GH19, GT84, and PL14 were enriched in $\mathrm{BE}(+)$ calves whereas the dominance of CE14 and GH89 was found in $\mathrm{BE}(-)$ calves compared to their counterparts (Table 4). The predominance of 11 CAZymes were divided by $\mathrm{PE}$ inoculation. However, dominance of those CAZymes in each type of microbial inoculation were not observed in their corresponded inoculums. 
Table 3 Exclusive network statistics by BE or PE

\begin{tabular}{|c|c|c|c|c|}
\hline \multirow[t]{2}{*}{ Network measurements } & \multicolumn{4}{|l|}{ Microbial inoculations } \\
\hline & $\mathrm{BE}(+)$ & $\mathrm{BE}(-)$ & $\mathrm{PE}(+)$ & $\mathrm{PE}(-)$ \\
\hline Total nodes & 137 & 106 & 137 & 128 \\
\hline Total edges & 260 & 123 & 199 & 194 \\
\hline Positive & 193 & 77 & 132 & 136 \\
\hline Negative & 67 & 46 & 67 & 58 \\
\hline Positive (\%) & 74.231 & 62.602 & 66.332 & 70.103 \\
\hline Negative (\%) & 25.769 & 37.398 & 33.668 & 29.897 \\
\hline No. of exclusive nodes & MD: 70; Arc: 4; Bac: 25 & MD: 13; Arc: 1; Bac: 8 & MD: 27; Arc: 2; Bac: 8 & MD: 48; Arc: 2; Bac: 21 \\
\hline Abundance of exclusive node (\%) & $\begin{array}{l}\text { MD: } 6.462 \% \\
\text { Arc: } 24.597 \% \\
\text { Bac: } 21.120 \%\end{array}$ & $\begin{array}{l}\text { MD: } 0.418 \% \\
\text { Arc: } 0.549 \% \\
\text { Bac: } 1.569 \%\end{array}$ & $\begin{array}{l}\text { MD: } 0.857 \% \\
\text { Arc: } 9.485 \% \\
\text { Bac: } 1.441 \%\end{array}$ & $\begin{array}{l}\text { MD: } 5.084 \% \\
\text { Arc: } 67.380 \% \\
\text { Bac: } 14.901 \%\end{array}$ \\
\hline Network diameter & 16 & 13 & 16 & 12 \\
\hline Graph density & 0.028 & 0.022 & 0.021 & 0.024 \\
\hline Modularity & 0.675 & 0.815 & 0.744 & 0.706 \\
\hline No. of communities & 17 & 16 & 22 & 22 \\
\hline Average clustering coefficient & 0.301 & 0.117 & 0.306 & 0.268 \\
\hline Average path length & 5.843 & 5.741 & 5.810 & 4.627 \\
\hline Best 'Authority' node & $\begin{array}{l}\text { M00022 } \\
\text { Prevotella }\end{array}$ & $\begin{array}{l}\text { M00184 } \\
\text { Sodaliphilus }\end{array}$ & $\begin{array}{l}\text { M00049 } \\
\text { Desulfobulbus }\end{array}$ & $\begin{array}{l}\text { M00014 } \\
\text { Ligilactobacillus }\end{array}$ \\
\hline Best 'Eigenvector centrality' node & $\begin{array}{l}\text { M00022 } \\
\text { Prevotella }\end{array}$ & $\begin{array}{l}\text { M00184 } \\
\text { Sodaliphilus }\end{array}$ & $\begin{array}{l}\text { M00049 } \\
\text { Desulfobulbus }\end{array}$ & $\begin{array}{l}\text { Mo0014 } \\
\text { Ligilactobacillus }\end{array}$ \\
\hline
\end{tabular}

$\mathrm{BE}$, bacterial-enriched rumen fluid; PE, protozoal-enriched rumen fluid; MD, KEGG modules; Arc, archaeal genera; Bac, bacterial genera

\section{Major active bacterial and archaeal genera significantly correlated to the various fermentation and animal measurements}

Correlation analysis between the relative abundance of major active bacterial and archaeal genera and rumen fermentation or animal performance measurements showed various significant associations (correlation coefficient, $|r| \geq 0.6 ; P<0.05$ ) (Fig. 7). Particularly for the different measurements in our previous study [15], three bacterial genera (Corynebacterium, Lactobacillus, and Limosilactobacillus) were positively correlated with body weight. Butyrivibrio was positively correlated to three of the animal measurements (hip width, paunch girth, and papillae length). Treponema was negatively correlated to total volatile fatty acids (VFA) concentration but positively correlated to ruminal $\mathrm{pH}$. A known butyrate producer, Megasphaera was positively correlated to the molar proportion of butyrate which were significantly higher in $\mathrm{PE}(-)$ calves than that of $\mathrm{PE}(+)$ calves. Even though the measurements were not differed by microbial inoculations, Desulfovibrio and Ereboglobus were negatively correlated to papillae counts from dairy calves.

\section{Discussion}

Dairy cattle organic matter utilization is inextricably linked with the microbes resident in the rumen. While the microbial community composition is influenced by the environment that the calves inhabit in early life, modifying the microbes to affect a desired productive phenotype could result in benefits for farmers. The established microbial community composition has proved to be resilient toward intended manipulation [1, 2, 38]. For this reason, we and others [11-15] have begun evaluating the possibility of intervening in microbial establishment to direct the community composition by early life inoculation. In this study, the microbial and functional shifts by pre-weaning microbial inoculations (BE and $\mathrm{PE}$ ) were analyzed by RNA-seq which enables the investigation of active transcript features in the rumen microbiome of post-weaned dairy calves.

\section{Differential bacterial taxa between RNA-seq and 16S amplicon-seq}

Among the shared major phyla and genera between two analytic pipelines, the relative abundances of Elusimicrobia, Fibrobacteres, Planctomycetes, Spirochaetes, and Butyricimonas, Fibrobacter, Oscillibacter, Selenomonas, Treponema were at least tenfold higher in RNA-seq compared to $16 \mathrm{~S}$ amplicon-seq and those taxa might be metabolically active at least in post-weaned dairy calves. In a previous study comparing three different microbiota datasets in beef steers [17], the relative abundance of Bacteroidetes, represented by the most abundant ruminal genus, Prevotella, was greater in the $16 \mathrm{~S}$ amplicon-seq 


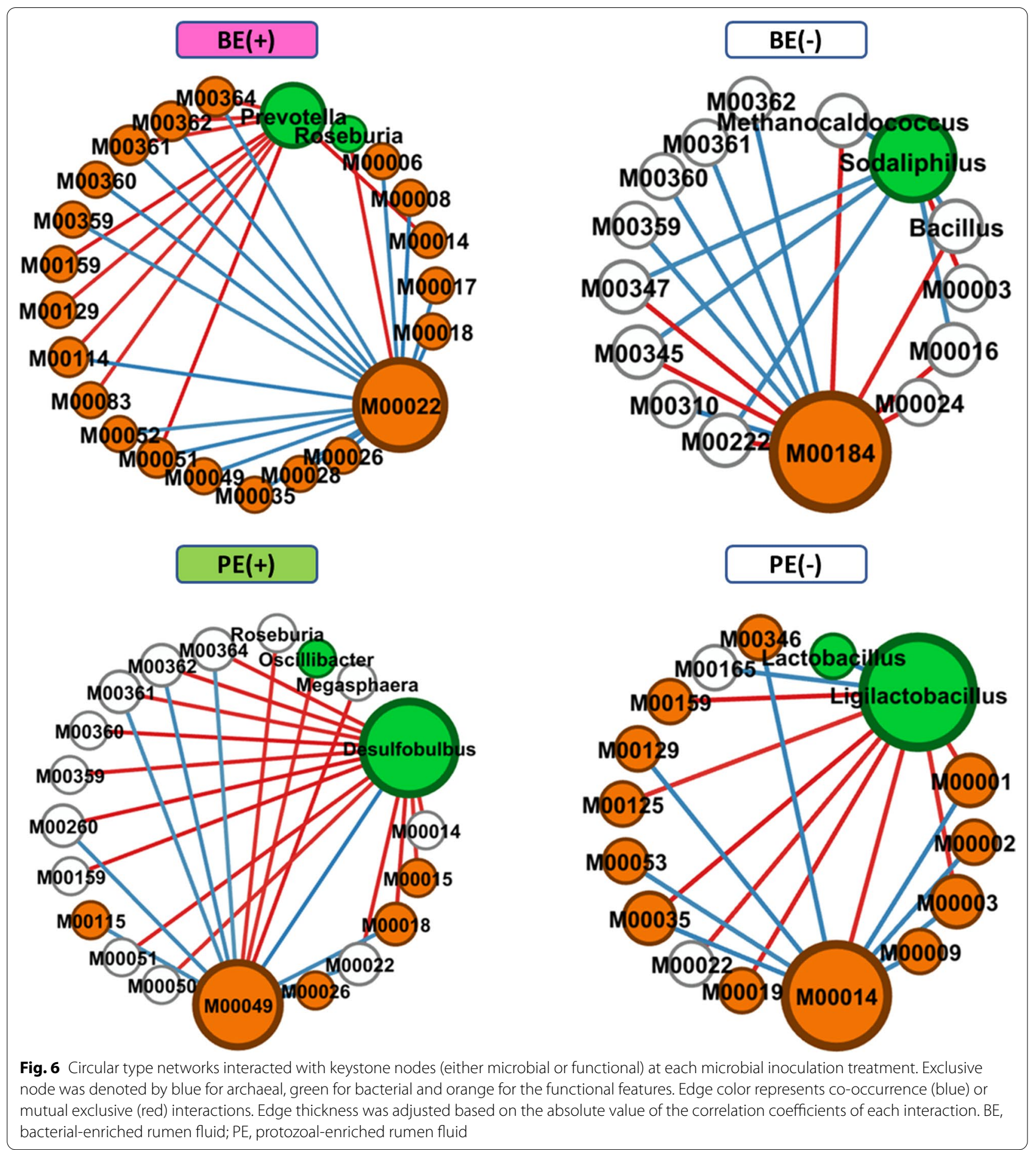

dataset compared to that of RNA-seq. Although Prevotella is the predominant ruminal bacterial genus in a variety of ruminal conditions [39-41], its relative abundance was 5.41-fold lower in RNA-seq datasets compared to that of $16 \mathrm{~S}$ amplicon-seq in this study. Based on these results, we speculate that their metabolic contribution to the overall rumen microbiome might have been overstated. However, longitudinal study based on the RNAseq pipeline will be necessary to further evaluate their fluctuation patterns in different dietary conditions particularly in adult dairy cattle. 
Table 4 Differentially abundant CAZymes by microbial inoculations

\begin{tabular}{|c|c|c|c|c|c|c|}
\hline \multirow[t]{2}{*}{ Dominance } & \multirow[t]{2}{*}{ CAZymes } & \multicolumn{2}{|c|}{$\begin{array}{l}\text { Relative abundance } \\
(\%)^{*}\end{array}$} & \multirow[t]{2}{*}{ SEM } & \multirow[t]{2}{*}{$P$-value } & \multirow[t]{2}{*}{ Known activities } \\
\hline & & $\mathrm{BE}(+)$ & $\mathrm{BE}(-)$ & & & \\
\hline \multirow[t]{3}{*}{$\mathrm{BE}(+)$} & GH19 & 0.010 & 0 & 0.003 & 0.031 & chitinase (EC 3.2.1.14) \\
\hline & GT84 & 0.053 & 0.004 & 0.013 & 0.009 & cyclic $\beta$-1,2-glucan synthase (EC 2.4.1.-) \\
\hline & PL14 & 0.067 & 0.013 & 0.011 & 0.010 & alginate lyase (EC 4.2.2.3) \\
\hline \multirow[t]{2}{*}{$\mathrm{BE}(-)$} & CE14 & 0.195 & 0.310 & 0.032 & 0.041 & $\begin{array}{l}\text { N-acetyl-1-D-myo-inosityl-2-amino-2-deoxy-a-D- } \\
\text { glucopyranoside deacetylase }\end{array}$ \\
\hline & GH89 & 0.147 & 0.242 & 0.026 & 0.023 & a-N-acetylglucosaminidase \\
\hline \multirow[t]{2}{*}{ Dominance } & CAZymes & \multicolumn{2}{|c|}{$\begin{array}{l}\text { Relative abundance } \\
(\%)\end{array}$} & SEM & $P$-value & Known activities \\
\hline & & $\mathrm{PE}(+)$ & $\mathrm{PE}(-)$ & & & \\
\hline \multirow[t]{5}{*}{$\mathrm{PE}(+)$} & GH16 & 0.314 & 0.182 & 0.038 & 0.041 & xyloglucan:xyloglucosyltransferase (EC 2.4.1.207) \\
\hline & GH39 & 0.363 & 0.253 & 0.056 & 0.028 & $\beta$-xylosidase (EC 3.2.1.37) \\
\hline & GH142 & 0.125 & 0.054 & 0.017 & 0.023 & $\beta$-L-arabinofuranosidase (EC 3.2.1.185) \\
\hline & PL10 & 0.121 & 0.032 & 0.017 & 0.006 & pectate lyase (EC 4.2.2.2) \\
\hline & PL33 & 0.051 & 0.015 & 0.008 & 0.006 & hyaluronate lyase (EC 4.2.2.1) \\
\hline \multirow[t]{6}{*}{$\mathrm{PE}(-)$} & CBM2 & 0.011 & 0.098 & 0.019 & 0.033 & cellulose-binding domain \\
\hline & CE11 & 0.662 & 0.991 & 0.061 & 0.007 & UDP-3-0-acyl N-acetylglucosamine deacetylase (EC 3.5.1.108) \\
\hline & GH92 & 0.574 & 0.959 & 0.082 & 0.010 & mannosyl-oligosaccharide a-1,2-mannosidase (EC 3.2.1.113) \\
\hline & $\mathrm{GH} 130$ & 0.416 & 0.864 & 0.084 & 0.008 & 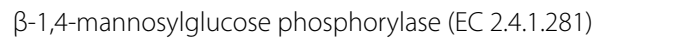 \\
\hline & GH133 & 0.861 & 1.161 & 0.067 & 0.023 & amylo-a-1,6-glucosidase (EC 3.2.1.33) \\
\hline & GT4 & 2.064 & 2.602 & 0.097 & 0.005 & sucrose synthase (EC 2.4.1.13) \\
\hline
\end{tabular}

Only the CAZymes which have over $0.01 \%$ average relative abundance in at least one of the treatments $[\mathrm{BE}(+), \mathrm{BE}(-), \mathrm{PE}(+), \mathrm{PE}(-)]$ were statistically analyzed by $\mathrm{LEfSe}$ $B E$, bacterial-enriched rumen fluid; $P E$, protozoal-enriched rumen fluid

${ }^{*}$ Calculated based on the normalized CAZymes count tables

The lack of differential relative abundance of Firmicutes between two analytic pipelines was consistent with previous comparison. However, the result on the abundance of Proteobacteria between the two methods was contrasting. In this study, the abundance of Proteobacteria represented by four genera, Desulfobulbus, Desulfovibrio, Campylobacter, and Succinivibrio, was more predominant in RNA-seq datasets $(0.93 \%$ in RNA-seq vs. $0.21 \%$ in $16 \mathrm{~S}$ amplicon-seq). It is plausible that differing diets provided to both dairy and beef cattle differentially affected the activity of a variety of bacterial taxa. Furthermore, predominance of several fibrolytic bacterial genera (e.g., Fibrobacter, Clostridium, and Ruminococcus) in RNA-seq datasets could be used as a potential barometer to evaluate fibrolytic activities in dairy cattle using metatranscriptomic approaches. Additionally, the taxa uniquely identified by RNA-seq, if those taxa have not been widely studied in the rumen, would fill the blind spots of $16 \mathrm{~S}$-based methods.

\section{Microbial inoculations affect the presence and absence of taxa distributions}

The active bacterial and archaeal genera exclusively detected in either the inoculum or inoculum-treated calves were minor taxa based on the relative abundance cutoff. This reveals that an active core of microbiota in the rumen liquid were shared among adult rumen fluid, microbial inoculum treated and non-treated calves. This may have resulted in similar animal phenotypes and fermentation by microbial inoculations.

\section{Differential bacterial taxa by microbial inoculations}

Proteobacteria was the only phylum altered by microbial inoculations, specifically by PE. This phylum has been known as major bacterial group in ruminants and particularly dominant in young ruminants $[4,41]$. Lower abundance of this phylum by PE might be directly triggered by inoculation of adult rumen fluid which was predominated by Bacteroidetes and Firmicutes. Or rumen fluid inoculation facilitated the transition of rumen microbial community of dairy calves to that of less proteobacterial-dominated which has been noticed by longitudinal observation in young ruminants $[4,5]$. 


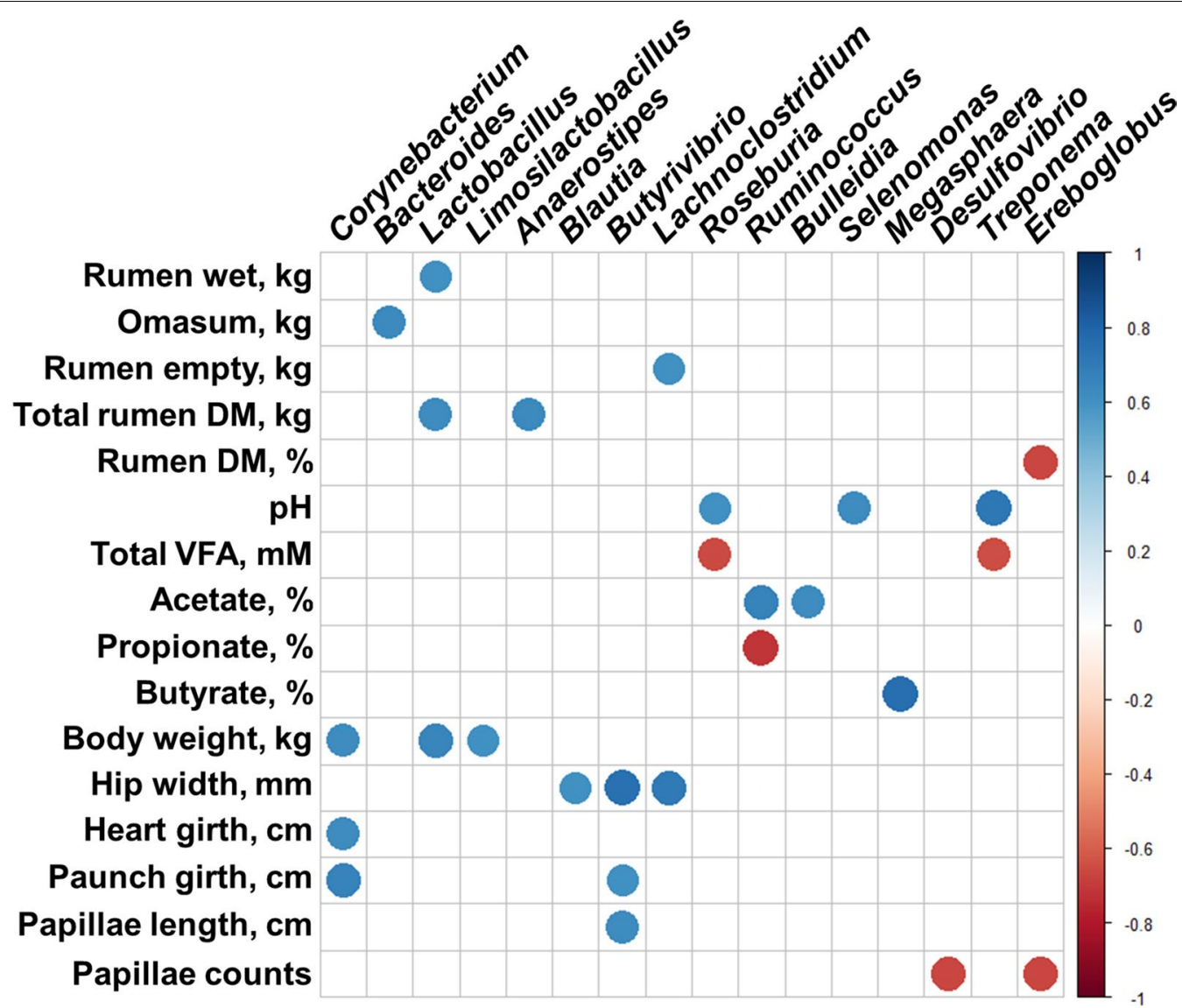

Fig. 7 Correlation between animal measurements with the relative abundance of major active bacterial and archaeal genera (occupying over $0.01 \%$ average relative abundance in at least one of the treatments). Only strong Pearson correlation coefficients $(P \leq 0.05 ;|r| \geq 0.6)$ were shown on the plot. The correlation coefficients were based both on the size and intensity of the color based on the color key on the right side

Bifidobacterium is not usually considered as major genus in adult rumen [42] and the abundance of this genus in $\mathrm{BE}(+)$ might be lowered by the establishment of normal microflora transferred from adult rumen fluid [9]. Among the bacterial genera predominant in $\mathrm{BE}(-)$ calves, Anaerostipes might contribute to butyrate production [43] in the rumen. However, there were no phenotypic differences found in their predominance between $\mathrm{BE}(-)$ and $\mathrm{BE}(+)$ calves.

$\mathrm{PE}(+)$ calves were dominant in Eubacterium and this genus containing the species of cellulose- and hemicellulose-degrading culturable rumen bacteria [31] and has been isolated from the bovine rumen [44]. Its predominance indicates an increase in fiber digestion. Desulfobulbus produces acetate as the major fermentation products of mono- and disaccharide fermentation [45]. Their predominance in $\mathrm{PE}(+)$ calves might affect the higher total ruminal VFA concentration compared to that of the $\mathrm{PE}(-)$ calves. A greater relative abundance of two butyrate producing bacteria might have resulted in the increase of the butyrate production in the $\mathrm{PE}(-)$ calves expecting to show better growth of rumen epithelial cells, papillae and development of the rumen $[3,46]$. However, as noticed in BE treatment, no phenotypic differences were corresponded to the greater butyrate molar proportion in the $\mathrm{PE}(-)$ calves.

Roseburia is a strong butyrate producer due to its ability to ferment starch [47]. The lactate-utilizing bacteria, Megasphaera, dominant in $\mathrm{PE}(-)$ calves also contributed to the greater butyrate molar proportion by converting lactate to butyrate $[48,49]$. Previous defaunation studies demonstrated that ruminal defaunation was negatively associated with butyrate molar proportion [50]. Only one calf in this study harbored holotrichs that are able to produce butyrate [51], whereas butyrate production by entodiniomorphs, the predominant type of ruminal protozoa, is not well-known. In addition to the negative correlation between NDF digestibility and butyrate proportions in defaunated animals [50], differed butyrate proportion by $\mathrm{PE}$ inoculation was related to the fiber digestibility and the composition of the metabolically active microbes varied in each treatment. 
Although, there were no differed archaeal taxa by microbial inoculations in this study, the overwhelming abundance of Methanocaldococcus in the active rumen microbiome of dairy calves in this study, in addition to the observance of its predominance in rumen wall by using metatranscriptome sequencing [52], needs to be further studied in terms of the actual contribution by this hydrogenotrophic methanogen to the overall rumen methane production.

\section{Functional distribution}

KEGG orthologs were predicted from the putative mRNAs of ruminal microbes. We extracted functional annotations from the blastx best hit outputs since the other metabolic pathway profiling tool (e.g., HUMAnN2) only mapped $1.60 \%$ of the reads to the known functions (data not shown). This could be due to the limited availability of functional genomic information of ruminal microbes [53].

For DNA-based methods, the differential microbial community or individual taxa abundances did not alter the microbial functions due to the functional redundancies among rumen microbes [54]. However, we observed that the overall microbial functional distribution was affected quantitatively by $\mathrm{BE}$ inoculation and qualitatively by PE inoculation. Even though the adult rumen fluid inoculation didn't change the overall ruminal bacterial communities, population shifts of the active microbiome and newly transferred microbial functions might result in the slightly altered animal performance and fermentations profiles of dairy calves. More distinguishable phenotypic changes by $\mathrm{PE}$ rather than by $\mathrm{BE}$ might result from the establishment of rumen protozoa, which affects a variety of microbial interactions and their symbiotic relationships.

\section{Differential individual functional feature abundances}

Among the analyzed fermentation characteristics, only ruminal $\mathrm{pH}$ was tended to be lower in $\mathrm{BE}(+)$ calves compared to that of $\mathrm{BE}(-)$ calves [15]. Two KEGG modules (M00006 and M00166) from $\mathrm{BE}(+)$ calves that participate in the formation of glyceraldehyde-3-phostphate via conversion of glucose to pyruvate may have contributed to a decrease in $\mathrm{pH}$ through the increased production of hydrogen [55].

The relative abundance of two amino acids biosynthesis modules (M00015 and M00020) was greater in $\mathrm{PE}(+)$ calves than that of $\mathrm{PE}(-)$ calves but this differential functional abundance did not result in increased total free amino acid concentrations and molar proportions of isobutyrate in $\mathrm{PE}(+)$ calves.
Spermidine and putrescine are synthesized from arginine [56] and possibly related to handling oxidative stress in the rumen [57]. The transport system of those polyamines was differentially abundant by both the $\mathrm{BE}$ and PE treatment and this is in agreement with the result of previous repeated adult rumen fluid treatment study with male calves [8].

\section{Exclusive microbial and functional networks by microbial inoculations}

The overall interactions within the rumen microbiome, which is represented by the abundance of major bacterial and archaeal genera and KEGG modules, were compared between microbial inoculation treatments. Shikimate pathway module (M00022) was the keystone function based on the centrality measurements within the exclusive networks in $\mathrm{BE}(+)$ calves. Together with the differentially enriched module related to pentose phosphate pathway (M00006) in $\mathrm{BE}(+)$ calves, shikimate pathway links between carbohydrate metabolism and aromatic amino acids biosynthesis and is a strong indicator of anabolic processes in the rumen microbiome $[58,59]$. Although, none of the exclusive positive interactions were found with phenylalanine, tyrosine and tryptophan biosynthesis pathway modules, shikimate pathway positively associated with carbohydrate pathways and many of biosynthesis pathways including vitamin, ribonucleotides, aminoacyl-tRNA, nucleotide sugars, and isoprenoid. This might be associated with the greater microbial protein synthesis and its growth triggered by $\mathrm{BE}$ inoculation, but they did not result in better fermentative and animal performances for $\mathrm{BE}(+)$ calves. Whereas, keystone bacterial genus, Prevotella showed negative associations with many of aforementioned biosynthesis pathway modules. Negative associations directly or indirectly related to the biosynthesis pathways (M00083, M00114, M00129, M00159, M00361, M00362, and M00364) with this keystone bacterial node implied the potential inefficient biosynthetic processes in Prevotella-dominant rumen microbiome of dairy calves.

In $\mathrm{BE}(-)$ calves, M00184 and Sodaliphilus were the keystone nodes within the exclusive network. The keystone nodes showed opposite associations particularly to the hydrogenotrophic methanogenesis pathway (M00347) and the abundance of Methanocaldococcus. M00184 is related to archaeal function [60] but its negative association with archaeal genus and methanogenesis pathway module need to be further studied in the context of archaeal transcription mechanism. Sodaliphilus, which was firstly described within pig microbiome [61], has been poorly characterized within rumen microbiome. Since its genome lacks genes encoding multiple glycolytic proteins [61] and hydrogenase in addition to 
its requirement of co-cultivation for better growth, the exclusive positive interaction with hydrogenotrophic methane production pathway might be indirect. Methane production measurements will be required to demonstrate the capacity of this exclusive networks in $\mathrm{BE}(-)$ calves.

Keystone nodes (M00049 and Desulfobulbus) in the exclusive network of $\mathrm{PE}(+)$ calves were positively associated each other and this interaction need to be understood within protozoa-harboring rumen microbiome. Purine metabolism is related to the cell growth and death [62], and this might be associated with bacterial predation activity of rumen protozoa inoculated by PE. In addition to its differential abundance in $\mathrm{PE}(+)$ calves, sulfate-reducing Desulfobulbus and its association with rumen protozoa is yet to be determined. Negative association with protein synthesis related pathway modules might be also related to having rumen protozoa particularly dominated by bacterivory entodiniomorphs [63].

Glucuronate pathway module (M00014) was the keystone functional module in the $\mathrm{PE}(-)$ calves positively interacted with many of the carbohydrate metabolisms (e.g., glycolysis, gluconeogenesis, citrate cycle) and formaldehyde assimilation module (M00346), which drives by methanotrophic bacteria to oxidize methane and methanol to formaldehyde, producing more hydrolytic products including pyruvate and methane [64]. However, these exclusive associations did not lead to fermentative responses beneficial to $\mathrm{PE}(-)$ calves. In addition to finding a greater number of enriched CAZymes in $\mathrm{PE}(-)$ calves compared to those of $\mathrm{PE}(+)$, the microbiome of $\mathrm{PE}(-)$ calves was associated with more complexed carbohydrate metabolism which usually linked to the inefficient utilization of feed by host animals [65, 66]. Ligilactobacillus was a keystone bacterial genus in the exclusive network of $\mathrm{PE}(-)$ calves. This genus is positively associated with another lactic acid bacteria, Lactobacillus. The occurrence of those probiotic bacterial genera, which enhance the gut health [67], might contribute to numerically better incidence of health measurements in $\mathrm{PE}(-)$ calves.

\section{Active bacterial genera correlated with fermentation and animal measurements}

Lactic acid bacteria, represented by the strains of Lactobacillus spp., has been widely used as probiotics to enhance the growth and health of young ruminants by preventing the colonization of pathogenic bacteria and boosting immune response [68]. Although the ruminal abundance of Lactobacillus is only numerically higher in inocula-treated calves, strong correlation between its abundance and body weight is plausible. Limited information is available for Corynebacterium but only mixed result was reported on the linkage between its jejunal abundance and feed efficiency of beef steers [69].

Butyrate is able to stimulate rumen papillae growth and development [70, 71]. Even though both the BE and PE didn't show different papillae length, correlation analyses demonstrated the positive correlation between the relative abundance of major butyrate producer, Butyrivibrio, and papillae length together with the hip width and paunch girth in post-weaned dairy calves. The relative abundances of two Desulfovibrio and Ereboglobus were negatively correlated with papillae counts. Increased abundance of sulfate reducing bacteria has been linked to the decreased integrity of rumen epithelium due to the $\mathrm{H}_{2} \mathrm{~S}$ production [72]. Ereboglobus is known as pectinolytic bacteria, and its abundance positively correlated to the acetate and propionate production [73] which inversely affected butyrate proportion in the rumen. Those association patterns found in active microbiome of dairy calves analyzed by using RNA-seq was quite different with those found in our previous amplicon sequencing based datasets.

\section{Conclusions}

RNA-seq allows us to understand what is actively transcribed by the rumen microbiome by analyzing both the coding and non-coding RNA species. Compared to our previous results from both the pre- and post-weaned calves analyzed by $16 \mathrm{~S}$ ampliconseq approaches, differentially abundant, active microbial taxa and functions analyzed by RNA-seq showed reliable connections with different fermentation characteristics and animal development measurements by adult rumen fluid inoculations. Interestingly, both the $\mathrm{BE}$ and $\mathrm{PE}$ inocula affected the quantitative and qualitative overall microbial functional distributions, respectively without microbial community differences. In addition to the minor transfer of adult, rumen-derived bacterial taxa into dairy calves, active microbial and functional abundances and network differences might be responsible for the fermentation and performance characteristics of dairy calves during and after inoculations. Future research should focus on the contents from the other compartments of the gastrointestinal tracts from a metatranscriptomics approach, which has the potential to better elucidate undetermined biological factors affected by microbial inoculations in dairy calves.

\section{Abbreviations}

$16 S$ amplicon-seq: 16S rRNA gene amplicon sequencing; BE: Bacterialenriched rumen fluid; CAZymes: Carbohydrate-active enzymes; KAAS: KEGG Automatic Annotation Server; KEGG: Kyoto Encyclopedia of Genes and Genomes; CBM: Carbohydrate-binding modules; CE: Carbohydrate esterases; CoDiNA: Co-expression Differential Network Analysis; GH: Glycosyl hydrolases; 
GT: Glycosyl transferases; PE: Protozoal-enriched rumen fluid; PERMANOVA: Permutational multivariate analysis of variance; PL: Polysaccharide lyases; RIN RNA integrity number; SEM: Standard error of the mean; VFA: Volatile fatty acids.

\section{Supplementary Information}

The online version contains supplementary material available at https://doi. org/10.1186/s42523-021-00142-z.

Additional file 1: Fig. S1. Analytic workflow of metatranscriptomics used in this study.

Additional file 2: Table S1. Calf health assessments. Table S2. RNA sequence statistics in inoculated samples. Table S3. RNA sequence statistics in inocula. Table S4. Alpha diversity measurements of bacterial and archaeal genera in microbial inocula.

Additional file 3: Fig. S2. NMDS plot shows the distribution of bacterial genera which were detected by both the 165 amplicon- and RNA-seq. Plots were drawn based on the Bray-Curtis distance matrices and significance was calculated using PERMANOVA test implemented in Vegan.

Additional file 4: Fig. S3. Principal coordinates analysis (PCOA) plot based on Bray-Curtis and Jaccard distance matrices representing overall active rumen archaeal microbiota at the genus level in the liquid fraction of dairy calves differed by microbial inoculations with two types of inocula (BE and PE). BE, bacterial-enriched rumen fluid; PE, protozoal-enriched rumen fluid.

Additional file 5: Fig S4. Exclusive co-occurrence and mutual exclusion microbial network by microbial inoculations based on the relative abundance of archaeal and bacterial genera and KEGG modules. Exclusive node was denoted by blue for archaeal, green for bacterial and orange for the functional features. Keystone node was marked as asterisks selected based on the authority and eigenvector centrality measurements within each exclusive network. Edge color represents co-occurrence (blue) or mutual exclusive (red) interactions. Edge thickness was adjusted based on the absolute value of the correlation coefficients of each interaction. BE, bacterial-enriched rumen fluid; PE, protozoal-enriched rumen fluid.

\section{Acknowledgements}

We acknowledge the excellent work of Dairy Forage Research Center farm staff for animal care and Rebecca Shilts and Ali Pelletier for assisting with sample collection. We also thank Dr. Garret Suen, Madison Cox, and Joseph Skarlupka of the Suen Lab for their assistance. We thank Robby Weyker and personnel at the Meat Science and Muscle Biology Laboratory for assistance with animal sacrifice and processing. This research was supported in part by an appointment to the Agricultural Research Service (ARS) Research Participation Program administered by the Oak Ridge Institute for Science and Education (ORISE) through an interagency agreement between the U.S. Department of Energy (DOE) and the U.S. Department of Agriculture (USDA). ORISE is managed by ORAU under DOE contract number DE-SC0014664. All opinions expressed in this paper are the author's and do not necessarily reflect the policies and views of USDA, DOE, or ORAU/ORISE.

\section{Authors' contributions}

LC and GZ conceived and designed the study. LC, WR, and GZ conducted the experiment and analyzed the animal data. TP and WL did the metatranscriptomic analysis. TP and LC drafted the manuscript. GZ and WL reviewed and edited the manuscript. All the authors read and approved the manuscript.

\section{Funding}

This research was supported by funding from the USDA, Agricultural Research Service under National Program 101 Food Animal Production. This research used resources provided by the SCINet project of the USDA Agricultural Research Service, ARS project number 0500-00093-001-00-D. Mention of any trademark or proprietary product in this manuscript does not constitute a guarantee or warranty of the product by the USDA or the Agricultural Research Service and does not imply its approval to the exclusion of other products that also may be suitable. USDA is an equal opportunity provider and employer.
Availability of data and materials

The raw RNA reads generated for this study can be found in NCBI Sequence Read Archive, PRJNA454463.

\section{Declarations}

Ethics approval and consent to participate

The animal study was reviewed and approved by University of Wisconsin's Institutional Animal Care and Use Committee. The protocol was approved by the University of Wisconsin's Institutional Animal Care and Use Committee under the protocol A005829.

\section{Consent for publication}

Not applicable.

\section{Competing interests}

The authors declare that they have no competing interests.

\section{Author details}

${ }^{1}$ USDA-Agricultural Research Service, Dairy Forage Research Center, Madison, WI, USA. ${ }^{2}$ Department of Animal Science and Technology, Chung-Ang University, Anseong, Gyeonggi-do, Korea.

Received: 1 March 2021 Accepted: 27 October 2021

Published online: 05 January 2022

\section{References}

1. Weimer P, Stevenson D, Mantovani H, Man S. Host specificity of the ruminal bacterial community in the dairy cow following near-total exchange of ruminal contents. J Dairy Sci. 2010;93:5902-12.

2. Weimer PJ. Redundancy, resilience, and host specificity of the ruminal microbiota: implications for engineering improved ruminal fermentations. Front Microbiol. 2015;6:296.

3. Baldwin R, McLeod K, Klotz J, Heitmann R. Rumen development, intestina growth and hepatic metabolism in the pre-and postweaning ruminant. J Dairy Sci. 2004;87:E55-65.

4. Jami E, Israel A, Kotser A, Mizrahi I. Exploring the bovine rumen bacterial community from birth to adulthood. ISME J. 2013;7:1069-79.

5. Rey M, Enjalbert F, Combes S, Cauquil L, Bouchez O, Monteils V. Establishment of ruminal bacterial community in dairy calves from birth to weaning is sequential. J Appl Microbiol. 2014;116:245-57.

6. Yáñez-Ruiz DR, Abecia L, Newbold CJ. Manipulating rumen microbiome and fermentation through interventions during early life: a review. Front Microbiol. 2015;6:10.

7. Huws SAH, Creevey C, Oyama LB, Mizrahi I, Denman SE, Popova M, Muñoz-Tamayo R, Forano E, Waters SM, Hess M. Addressing global ruminant agricultural challenges through understanding the rumen microbiome: past, present and future. Front Microbiol. 2018;9:2161.

8. Bu D, Zhang X, Ma L, Park T, Wang L, Wang M, Xu J, Yu Z. Repeated inoculation of young calves with rumen microbiota does not significantly modulate the rumen prokaryotic microbiota consistently but decreases diarrhea. Front Microbiol. 2020;11:1403.

9. Yu S, Zhang G, Liu Z, Wu P, Yu Z, Wang J. Repeated inoculation with fresh rumen fluid before or during weaning modulates the microbiota composition and co-occurrence of the rumen and colon of lambs. BMC Microbiol. 2020;20:29.

10. Belanche A, Palma-Hidalgo J, Nejjam I, Jiménez E, Martín-García A, Yáñez-Ruiz D. Inoculation with rumen fluid in early life as a strategy to optimize the weaning process in intensive dairy goat systems. J Dairy Sci. 2020;103:5047-60.

11. Malmuthuge N, Griebel PJ. Taxonomic identification of commensal bacteria associated with the mucosa and digesta throughout the gastrointestinal tracts of preweaned calves. Appl Environ Microbiol. 2014;80:2021-8.

12. Ishaq SL. Wright A-DG: Insight into the bacterial gut microbiome of the North American moose (Alces alces). BMC Microbiol. 2012;12:212.

13. Jiao J, Huang J, Zhou C, Tan Z. Taxonomic identification of ruminal epithelial bacterial diversity during rumen development in goats. Appl Environ Microbiol. 2015:81:3502-9. 
14. Cersosimo LM, RadloffW, Zanton GI. Microbial inoculum composition and pre-weaned dairy calf age alter the developing rumen microbial environment. Front Microbiol. 2019;10:1651.

15. Park T, Cersosimo LM, Li W, Radloff W, Zanton Gl. Pre-weaning ruminal administration of differentially-enriched, rumen-derived inocula shaped rumen bacterial communities and co-occurrence networks of post-weaned dairy calves. Front Microbiol. 2021;12:311.

16. Gaidos E, Rusch A, llardo M. Ribosomal tag pyrosequencing of DNA and RNA from benthic coral reef microbiota: community spatial structure, rare members and nitrogen-cycling guilds. Environ Microbiol. 2011;13:1138-52

17. Li F, Henderson G, Sun X, Cox F, Janssen PH, Guan LL. Taxonomic assessment of rumen microbiota using total RNA and targeted amplicon sequencing approaches. Front Microbiol. 2016;7:10.

18. Kim Y-H, Toji N, Kizaki K, Kushibiki S, Ichijo T, Sato S. Effects of dietary forage and calf starter on ruminal $\mathrm{pH}$ and transcriptomic adaptation of the rumen epithelium in Holstein calves during the weaning transition. Physiol Genomics. 2016:48:803-9.

19. Li W, Edwards A, Riehle C, Cox M, Raabis S, Skarlupka J, Steinberger A, Walling J, Bickhart D, Suen G. Transcriptomics analysis of host liver and meta-transcriptome analysis of rumen epimural microbial community in young calves treated with artificial dosing of rumen content from adult donor cow. Sci Rep. 2019;9:790.

20. Li W, Gelsinger S, Edwards A, Riehle C, Koch D. Transcriptome analysis of rumen epithelium and meta-transcriptome analysis of rumen epimural microbial community in young calves with feed induced acidosis. Sci Rep. 2019;9:4744.

21. Dobin A, Davis CA, Schlesinger F, Drenkow J, Zaleski C, Jha S, Batut $P$, Chaisson M, Gingeras TR. STAR: ultrafast universal RNA-seq aligner. Bioinformatics. 2013;29:15-21.

22. Wood DE, Salzberg SL. Kraken: ultrafast metagenomic sequence classification using exact alignments. Genome Biol. 2014;15:R46.

23. Kopylova E, Noé L, Touzet H. SortMeRNA: fast and accurate filtering of ribosomal RNAs in metatranscriptomic data. Bioinformatics. 2012:28:3211-7.

24. Quast C, Pruesse E, Yilmaz P, Gerken J, Schweer T, Yarza P, Peplies J, Glöckner FO. The SILVA ribosomal RNA gene database project: improved data processing and web-based tools. Nucleic Acids Res. 2012;41:D590-6.

25. Li W, Gelsinger S, Edwards A, Riehle C, Koch D. Changes in meta-transcriptome of rumen epimural microbial community and liver transcriptome in young calves with feed induced acidosis. Sci Rep. 2019;9:1-12.

26. Watts SC, Ritchie SC, Inouye M, Holt KE. FastSpar: rapid and scalable correlation estimation for compositional data. Bioinformatics. 2019;35:1064-6.

27. Friedman J, Alm EJ. Inferring correlation networks from genomic survey data. PLoS Comput Biol. 2012;8:e1002687.

28. Gysi DM, Fragoso TM, Buskamp V, Almaas E, Nowick K. Comparing multiple networks using the Co-expression Differential Network Analysis (CoDiNA). arXiv preprint arXiv:180200828; 2018.

29. Bastian M, Heymann S, Jacomy M. Gephi: an open source software for exploring and manipulating networks. In: Third international AAAI conference on weblogs and social media (2009).

30. Callahan BJ, McMurdie PJ, Rosen MJ, Han AW, Johnson AJA, Holmes SP. DADA2: high-resolution sample inference from Illumina amplicon data. Nat Methods. 2016;13:581-3.

31. Dai X, Tian Y, Li J, Su X, Wang X, Zhao S, Liu L, Luo Y, Liu D, Zheng H. Metatranscriptomic analyses of plant cell wall polysaccharide degradation by microorganisms in cow rumen. Appl Environ Microbiol 2014:AEM. $03682-03614$

32. Buchfink B, Xie C, Huson DH. Fast and sensitive protein alignment using DIAMOND. Nat Methods. 2015;12:59.

33. Moriya Y, Itoh M, Okuda S, Yoshizawa AC, Kanehisa M. KAAS: an automatic genome annotation and pathway reconstruction server. Nucleic Acids Res. 2007;35:W182-5.

34. Douglas GM, Maffei VJ, Zaneveld J, Yurgel SN, Brown JR, Taylor CM, Huttenhower C, Langille MG. PICRUSt2: An improved and extensible approach for metagenome inference. BioRxiv 2019:672295.

35. Zhang $H$, Yohe $T$, Huang L, Entwistle S, Wu P, Yang Z, Busk PK, Xu Y, Yin Y. dbCAN2: a meta server for automated carbohydrate-active enzyme annotation. Nucleic Acids Res. 2018:46:W95-101.
36. Busk PK, Pilgaard B, Lezyk MJ, Meyer AS, Lange L. Homology to peptide pattern for annotation of carbohydrate-active enzymes and prediction of function. BMC Bioinform. 2017:18:1-9.

37. Segata N, Izard J, Waldron L, Gevers D, Miropolsky L, Garrett WS, Huttenhower C. Metagenomic biomarker discovery and explanation. Genome Biol. 2011;12:R60

38. Weimer PJ, Cox MS, de Paula TV, Lin M, Hall MB, Suen G. Transient changes in milk production efficiency and bacterial community composition resulting from near-total exchange of ruminal contents between highand low-efficiency Holstein cows. J Dairy Sci. 2017;100:7165-82.

39. Kittelmann S, Seedorf H, Walters WA, Clemente JC, Knight R, Gordon J, Janssen PH. Simultaneous amplicon sequencing to explore co-occurrence patterns of bacterial, archaeal and eukaryotic microorganisms in rumen microbial communities. PLoS ONE. 2013;8:e47879.

40. Petri RM, Schwaiger T, Penner GB, Beauchemin KA, Forster RJ, McKinnon $\mathrm{J}$, McAllister TA. Characterization of the core rumen microbiome in cattle during transition from forage to concentrate as well as during and after an acidotic challenge. PLoS ONE. 2013;8:e83424.

41. Henderson G, Cox F, Ganesh S, Jonker A, Young W, Collaborators GRC, Janssen PH. Rumen microbial community composition varies with diet and host, but a core microbiome is found across a wide geographical range. Sci Rep. 2015;5:14567.

42. Fonty G, Gouet P, Jouany J-P, Senaud J. Establishment of the microflora and anaerobic fungi in the rumen of lambs. Microbiology. 1987; 133:1835-43

43. Allen-Vercoe E, Daigneault M, White A, Panaccione R, Duncan SH, Flint HJ, O'Neal L, Lawson PA. Anaerostipes hadrus comb. nov., a dominant species within the human colonic microbiota; reclassification of Eubacterium hadrum Moore et al. 1976. Anaerobe. 2012;18:523-9.

44. Prins R, Van Vugt F, Hungate R, Van Vorstenbosch C. A comparison of strains of Eubacterium cellulosolvens from the rumen. Antonie Leeuwenhoek. 1972;38:153-61.

45. Boone DR, Goodfellow M, Rainey FA, Schleifer K-H, Vos P. Bergey's manual of systematic bacteriology: Volume 2: The Proteobacteria Part C The Alpha-, Beta-, Delta-, and Epsilonproteobacteria. New York: Springer; 2005

46. Song Y, Malmuthuge N, Steele MA, Guan LL. Shift of hindgut microbiota and microbial short chain fatty acids profiles in dairy calves from birth to pre-weaning. FEMS Microbiol Ecol. 2018;94:fix179.

47. Vos P, Garrity G, Jones D, Krieg NR, Ludwig W, Rainey FA, Schleifer K-H, Whitman W. Bergey's manual of systematic bacteriology: Volume 3: The Firmicutes. New York: Springer; 2011

48. Marounek M, Fliegrova K, Bartos S. Metabolism and some characteristics of ruminal strains of Megasphaera elsdenii. Appl Environ Microbiol. 1989;55:1570-3.

49. Henning P, Campbell A, Hagg F, Horn C. Effect of accelerated diet step-up rate on performance of feedlot steers dosed with Megasphaera elsdenii NCIMB 41125. Ruminant Physiology-Digestion, Metabolism and Effects of Nutrition on Reproduction and Animal Welfare Eds Chilliard, Y, Glasser, F, Faulconnieur, Y, Boucquier, Y, Veissier, I \& Doreau, M, Wageningen Academic Publishers 2009:78-79.

50. Newbold CJ, de la Fuente G, Belanche A, Ramos-Morales E, McEwan NR. The role of ciliate protozoa in the rumen. Front Microbiol. 2015;6:1313.

51. Yarlett N, Lloyd D, Williams A. Butyrate formation from glucose by the rumen protozoon Dasytricha ruminantium. Biochem J. 1985;228:187-92.

52. Mann E, Wetzels SU, Wagner M, Zebeli Q, Schmitz-Esser S. Metatranscriptome sequencing reveals insights into the gene expression and functional potential of rumen wall bacteria. Front Microbiol. 2018;9:43.

53. Li F, Neves AL, Ghoshal B, Guan L. Symposium review: Mining metagenomic and metatranscriptomic data for clues about microbial metabolic functions in ruminants. J Dairy Sci. 2018;101:5605-18.

54. Taxis TM, Wolff S, Gregg SJ, Minton NO, Zhang C, Dai J, Schnabel RD, Taylor JF, Kerley MS, Pires JC. The players may change but the game remains: network analyses of ruminal microbiomes suggest taxonomic differences mask functional similarity. Nucleic Acids Res. 2015;43:9600-12.

55. Ungerfeld EM. Metabolic hydrogen flows in rumen fermentation: Principles and possibilities of interventions. Front Microbiol. 2020;11:589.

56. Soda K. The mechanisms by which polyamines accelerate tumor spread. J Exp Clin Cancer Res. 2011;30:1-9.

57. Minois N, Carmona-Gutierrez D, Madeo F. Polyamines in aging and disease. Aging (Albany NY). 2011;3:716. 
58. Mackie R, White B. Gastrointestinal microbiology: volume 1 gastrointestinal ecosystems and fermentations. Berlin: Springer; 2012.

59. Kamke J, Kittelmann S, Soni P, Li Y, Tavendale M, Ganesh S, Janssen PH, Shi W, Froula J, Rubin EM. Rumen metagenome and metatranscriptome analyses of low methane yield sheep reveals a Sharpea-enriched microbiome characterised by lactic acid formation and utilisation. Microbiome. 2016;4:56.

60. Jun S-H, Reichlen MJ, Tajiri M, Murakami KS. Archaeal RNA polymerase and transcription regulation. Crit Rev Biochem Mol Biol. 2011:46:27-40.

61. Wylensek D, Hitch TC, Riedel T, Afrizal A, Kumar N, Wortmann E, Liu T, Devendran S, Lesker TR, Hernández SB. A collection of bacterial isolates from the pig intestine reveals functional and taxonomic diversity. Nat Commun. 2020;11:1-26.

62. Ribeiro DM, Salama A, Vitor AC, Arguello A, Moncau CT, Santos EM, Caja G, de Oliveira JS, Balieiro JC, Hernández-Castellano LE. The application of omics in ruminant production: a review in the tropical and sub-tropical animal production context. J Proteomics. 2020;1:103905.

63. Belanche A, de la Fuente G, Moorby JM, Newbold CJ. Bacterial protein degradation by different rumen protozoal groups. J Anim Sci. 2012:90:4495-504.

64. Lim JW, Park T, Tong YW, Yu Z. The microbiome driving anaerobic digestion and microbial analysis. Adv Bioenergy. 2020;5:1-14.

65. Shabat SKB, Sasson G, Doron-Faigenboim A, Durman T, Yaacoby S, Miller MEB, White BA, Shterzer N, Mizrahi I. Specific microbiome-dependent mechanisms underlie the energy harvest efficiency of ruminants. ISME J. 2016;10:2958-72.

66. Xue M-Y, Sun H-Z, Wu X-H, Liu J-X, Guan LL. Multi-omics reveals that the rumen microbiome and its metabolome together with the host metabolome contribute to individualized dairy cow performance. Microbiome. 2020;8:1-19.

67. Uyeno Y, Shigemori S, Shimosato T. Effect of probiotics/prebiotics on cattle health and productivity. Microbes Environ 2015:ME14176.

68. Lei Y, Zhang K, Guo M, Li G, Li C, Li B, Yang Y, Chen Y, Wang X. Exploring the spatial-temporal microbiota of compound stomachs in a pre-weaned goat model. Front Microbiol. 1846;2018:9.

69. Myer P, Wells J, Smith T, Kuehn L, Freetly H. Microbial community profiles of the jejunum from steers differing in feed efficiency. J Anim Sci. 2016;94:327-38.

70. Flatt W, Warner R, Loosli J. Influence of purified materials on the development of the ruminant stomach. J Dairy Sci. 1958;41:1593-600.

71. Sander E, Warner R, Harrison H, Loosli J. The stimulatory effect of sodium butyrate and sodium propionate on the development of rumen mucosa in the young calf. J Dairy Sci. 1959;42:1600-5.

72. Drewnoski M, Pogge D, Hansen S. High-sulfur in beef cattle diets: a review. J Anim Sci. 2014;92:3763-80.

73. Tegtmeier D, Belitz A, Radek R, Heimerl T, Brune A. Ereboglobus /uteus gen. nov. sp. Nov. from cockroach guts, and new insights into the oxygen relationship of the genera Opitutus and Didymococcus (Verrucomicrobia: Opitutaceae). Syst Appl Microbiol. 2018;41:101-12.

\section{Publisher's Note}

Springer Nature remains neutral with regard to jurisdictional claims in published maps and institutional affiliations.

Ready to submit your research? Choose BMC and benefit from:

- fast, convenient online submission

- thorough peer review by experienced researchers in your field

- rapid publication on acceptance

- support for research data, including large and complex data types

- gold Open Access which fosters wider collaboration and increased citations

- maximum visibility for your research: over $100 \mathrm{M}$ website views per year

At BMC, research is always in progress.

Learn more biomedcentral.com/submissions 